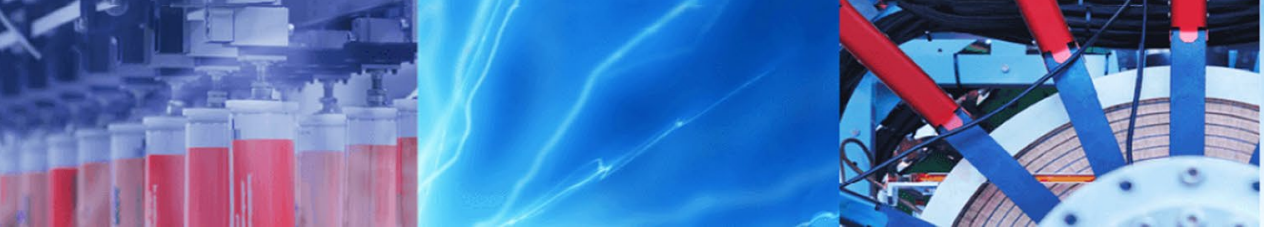

Research Article

\title{
Free vibration analysis of Timoshenko beams reinforced by BNNTs and a comparison with CNT-reinforced composite
}

\author{
Ilda Abdollahi' ${ }^{1}$. Mohammad Hossein Yas ${ }^{1}$
}

Received: 2 September 2019 / Accepted: 5 March 2020 / Published online: 13 March 2020

(c) Springer Nature Switzerland AG 2020

\begin{abstract}
This paper studies free vibration of composite beams reinforced by boron nitride nanotubes (BNNTs), lying on an elastic foundation. The beam matrix is taken as poly methyl methacrylate. The nanotubes have different layouts in the matrix (aligned and randomly oriented) and are also distributed through the beam thickness as one uniform and three different functionally graded distributions. A micromechanical model is applied to estimate the properties of BNNT-reinforced composite (BNNTRC). Equations of motion are achieved by means of Hamilton's principle as well as Timoshenko beam theory. Natural frequencies are gained by introducing generalized differential quadrature method to the equations. Investigating the influence of different parameters as diverse as nanotube volume fraction, nanotubes distribution type, the elastic foundation, boundary conditions and the beam's slenderness ratio on the natural frequency, reveals that any changes in aforementioned parameters has a significant impact on the natural frequency. Furthermore, the results for aligned BNNTRC beam are measured against the results of similar CNT-reinforced beam in the literature, illustrating that natural frequency of BNNTRC beam is lower than CNTRC beam. Finally, the comparison between natural frequencies of two BNNTRC beams with two different alignments of BNNTs, illustrates their different behavior due to different factors.
\end{abstract}

Keywords Boron nitride nanotube · BNNT reinforced composite - Timoshenko beam · Free vibration · Elastic foundation · Differential quadrature method

\section{Introduction}

After the identification of carbon nanotubes (CNTs) and studying their outstanding properties and usage in reinforcing materials, many investigations were conducted to produce nanotubes of other materials, having similar atomic structure to carbon [1]. As boron nitride has hexagonal atomic structure, like carbon, many experiments were carried out and led to producing boron nitride nanotubes (BNNTs) [2]. Understanding their superb properties has made them outstanding reinforcement candidates for composites, for they have small density and significantly high mechanical properties [3, 4]. BNNTs possess a high thermal conductivity and an extremely high Young's modulus about 1 Tpa. but slightly smaller than for CNTs
[5]. Thanks to their extreme resistance to oxidation, BNNTs are stable up to $900-950^{\circ} \mathrm{C}$, while CNTs start to burn at around $500^{\circ} \mathrm{C}$ (in air) [6]. The bonds between $\mathrm{B}-\mathrm{N}$ atoms in BNNTs enjoy a ionic character which makes the structure stronger than CNTs, and also more chemically resistant [7]. The composites reinforced by BNNTs benefit from high mechanical strength, thermal stability, chemical resistance and thermal conductivity as well as small thermal expansion coefficient [8]. Despite CNTs, which are conductive or semi-metallic based on their chirality, BNNTs are nonconductive materials independent of their chirality [9]. The most remarkable property of BNNTs in reinforcing polymer matrices is associated with this property, which saves the dielectric nature of polymer [10]. BNNT reinforced nanocomposites are suitable for a broad range of applications

Ilda Abdollahi, ilda89abd@gmail.com | 'Department of Mechanical Engineering, Razi University, Kermanshah, Iran. 
in optoelectronics, light-harvesting, piezoelectric applications and also reinforced structural materials for various usages in different industries [11]. In summary, due to better thermal and chemical stabilities in comparison with CNTs, BNNTs offer a wide range of applications for reinforcement of composite materials to be used at elevated temperatures in oxidizing environment. As insulating fillers of high thermal conductivity and mechanical strength, BNNTs are also suitable for dielectric purposes. That is the main reason why authors decided to carry out a painstaking research on vibration of BNNT reinforced nanocomposite beams and made a comparison with CNTRC beams.

Liew et al. [12] investigated mechanical analysis of functionally graded (FG) CNT reinforced composites (CNTRC), including static, dynamic and nonlinear analysis and provided a thorough overview on the recent advances on FG-CNTRCs based on a detailed review of researches. In another review Liew et al. [13] presented the latest progress in the modeling methods for mechanical properties of CNTRCs including atomistic, continuum mechanics and multiscale modeling, as well as mechanical analysis of FG-CNTRC structures as static, vibration, buckling and postbuckling analysis and clearly provided a picture of the mechanical modeling and properties of FG-CNTRCs as well as their composite structures. Yas and Tahouneh [14] used differential quadrature method to investigate 3-D free vibration behavior of a thick FG annular plate on paternak elastic foundation. Zhang [15] investigated the effect of in-plane forces on the vibration behavior of CNTRC skew plates using first-order shear deformation theory along with a mesh-free Improved Moving Least Square-Ritz (IMLS-R) method, considering two types of nanotube distribution as uniformly and functionally graded. In another study, Zhang [16] employed the first-order shear deformation plate theory to analyze buckling behavior of nanocomposite plates of polygonal planform reinforced by single-walled CNTs under in-plane loads, and used IMLS-R method to solve the governing eigenvalue equation. Ke et al. [17] conducted a research on the flexural vibration and elastic buckling of a cracked exponentially distributed FG beam subjected to various boundary conditions using Timoshenko beam theory. Using generalized differential quadrature method (GDQM), Yas and Samadi 18] analyzed free vibration and buckling of functionally graded CNT-reinforced Timoshenko beams, lying on an elastic foundation. Yan et al. [19] investigated dynamic behavior of edge-cracked exponentially distributed FG beams on an elastic foundation under the action of a moving load using Timoshenko beam theory. Liew et al. [20] conducted a review on layerwise theories for composite laminates and structures, representing the recent development of layerwise theories, their numerical implementation, and application in the analysis of composite laminated structures. Utilizing Reddy's higher-order shear deformation theory and taking advantage of IMLS-R method, Zhang and Selim [21] studied free vibration behavior of CNTRC functionally graded thick laminated composite plates, considering 4 different distributions for CNTs and analyzing the impact of different parameters on the vibration behavior. Shooshtari and Rafiee [22] used Euler-Bernoulli beam theory and von karman nonlinearity alongside Galerkin method to carry out a research on nonlinear forced vibration of functionally graded clamped beams. Based on molecular dynamic simulations, Cong and Lee [23] researched mechanical behavior of BNNT-Al composites, subjected to tensile loading and revealed significance effect of BNNTs volume fraction on enhancing mechanical properties of composites. The literature review found no reported research work on vibration analysis of nanocomposite beams reinforced by BNNTs and it is inferred that researches are mostly related to enhancing mechanical and thermal properties of BNNT reinforced nanocomposites with various materials taken as matrix as well as thorough researches on CNT-reinforced composites, including mechanical, vibrational and buckling analysis [24-30].

Therefore, the main goal of this research and the associated novelty was to carry out a painstaking and thorough investigation on free vibration behavior of polymer-based BNNT-reinforced composite beams. Factoring in that BNNTs are electrical insulator and they ensure saving the natural dielectric property of the polymer matrix, while considerably improving mechanical and thermal properties, and the fact that the dielectric property of polymer matrix is eliminated in CNT-reinforced composites [31, 32], in this paper a thorough comparison was also conducted between the free vibration behavior of these two types of nanotube-reinforced polymeric composites.

In this study, free vibration of a nanocomposite beam was investigated. During this investigation, a nanocomposite beam reinforced by single-walled BNNTs (SWBNNT) was considered to be lying on an elastic foundation and was also subjected to different boundary conditions. The nanotubes were assumed to be straight and perfect, with an aligned or a randomly oriented layout in the matrix and they were also considered to be distributed uniformly or functionally graded through the thickness direction. Employing a micromechanical model, the mechanical properties of BNNTRC beams were approximated and Timoshenko beam theory was applied to derive the governing equations, so as to factor in rotary inertia and transverse shear deformation. Applying generalized differential quadrature method to the governing equations of motion, equations were solved, natural frequencies were derived and ultimately they were compared with the similar ones of CNTRC beams in the literatures. 


\section{Material properties of BNNTRC beams}

The results of multi-scale FEM, leads to the conclusion that the BNNT and its inter-phase could be considered as an equivalent fiber. Because the polymer molecules are on the same size scale as the nanotubes, the interaction at the polymer/nanotube interface is highly dependent on the local molecular structure and bonding. At these small length scales, the lattice structures of the nanotube and polymer chains cannot be considered continuous, and the bulk mechanical properties can no longer be determined through traditional micromechanical approaches. It is proposed that the nanotube, the local polymer near the nanotube, and the nanotube/polymer interface can be modeled as an effective continuum fiber by using an equivalent-continuum modeling method [33]. Moreover, due to the high young's modulus and stiffness of nanotubes compared with most polymer matrices, nanotubes are treated as rigid fibers in the matrix [34]. Therefore, in order to predict the mechanical properties of the BNNT-polymer composite, an embedded BNNT in the matrix is considered as an equivalent long fiber [35]. The BNNTRC beams are considered to consist of SWBNNTs and an isotropic polymer matrix. The mechanical properties are calculated through a micromechanical model. In which the effective mechanical properties, such as Young's and shear modulus and Poisson's ratio of BNNTRC beams are estimated for two different BNNTs distribution types. As depicted in Fig. 1

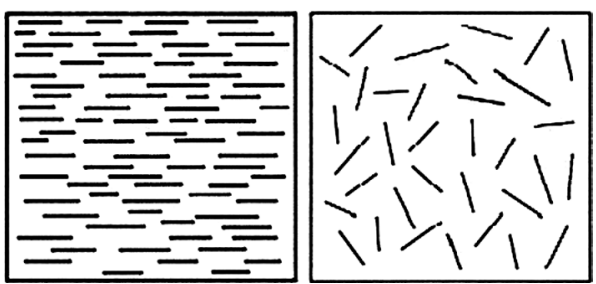

Fig. 1 BNNTs alignment in the matrix: aligned and randomly oriented left to right, respectively

Fig. 2 BNNTs distribution type through the beam thickness the first assumption is based on a unidirectional distribution of nanotubes and the other distribution is assumed to be randomly oriented through the matrix.

The mechanical properties of BNNTRC materials are derived from the Halpin-Tsai equations with the assumptions as fiber cross section is circular and a perfect bonding exists between the fibers and matrix and the matrix is free of voids, that BNNTs and the matrix satisfy these assumptions [36]. The elastic properties of BNNTRC beams of unidirectionally or randomly oriented BNNTs are thoroughly explained and calculated in "Appendix".

To investigate the effect of BNNTs distribution along the beam's thickness direction; one uniform distribution (UD) and three different functionally graded distributions (FG- $\Lambda, F G-\diamond$ and $F G-X)$ for nanotubes were considered, as shown in Fig. 2. Despite the constancy of BNNTs density, the volume fraction of BNNTs varies through the beam thickness based on the distribution type. The above-mentioned distributions of the BNNTs are described as:

$U D: \quad V_{b n n t}=V_{b n n t}^{*}$

FG- $\diamond: \quad V_{b n n t}=\left(2-4 \frac{|z|}{a}\right) V_{b n n t}^{*}$

$F G-\wedge: \quad V_{b n n t}=\left(1-\frac{2 z}{a}\right) V_{b n n t}^{*}$

FG-X: $\quad V_{b n n t}=4 \frac{|z|}{a} V_{b n n t}^{*}$

where $a$ indicates the beam thickness along z-axis direction, and $z$ denotes the distance of a hypothetical section that we are looking for the volume fraction distribution in it, and varies from $(-a)$ to $(+a) . \mathrm{V}_{\mathrm{bnnt}}^{*}$ is the BNNTs volume fraction and is expressed as:

$V_{b n n t}^{*}=\frac{W_{b n n t}}{W_{b n n t}+\left(\rho^{b n n t} / \rho^{m}\right)\left(1-W_{b n n t}\right)}$

where $W_{b n n t}$ stands for the mass fraction of nanotubes, and $\rho^{\text {bnnt }}$ and $\rho^{m}$ are the mass density of BNNTs and the matrix, respectively.
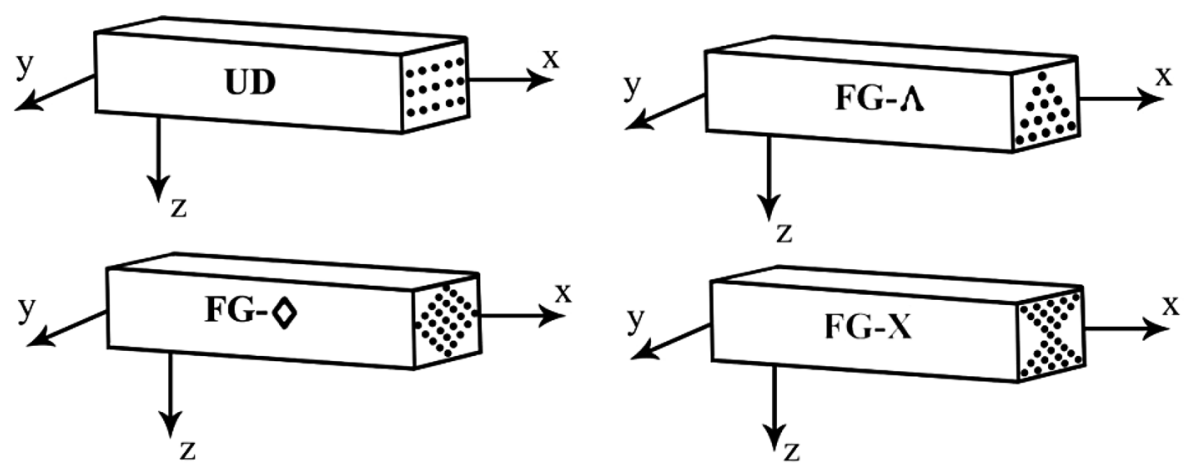


\section{Theory and formulations}

\subsection{Governing equations}

According to Timoshenko beam theory, the axial and transverse displacements of any point in the beam denoted by $\mathrm{U}(\mathrm{x}, \mathrm{z}, \mathrm{t})$ and $\mathrm{W}(\mathrm{x}, \mathrm{z}, \mathrm{t})$ respectively as follows: [37]

$U(x, z, t)=u_{0}(x, t)+z \Psi(x, t)$

$W(x, z, t)=w_{0}(x, t)$

where $u_{0}$ and $w_{0}$ are axial and transverse displacements at the mid-surface of the beam, and $\Psi$ denotes rotation of the section normal vector about the $y$ axis, and $t$ represents time.

The strain-displacements relations are expressed as:

$\varepsilon_{x}=\frac{d u_{0}}{d x}+z \frac{d \Psi}{d x}$

$\gamma_{x z}=\frac{d w_{0}}{d x}+\Psi$

Therein $\varepsilon_{x}$ and $\gamma_{x z}$ represent linear normal strain and shear strain respectively.

The normal and shear stress $\left(\sigma_{x}\right.$ and $\left.\tau_{x z}\right)$ are associated with normal and shear strain through linear elastic constitutive law as:

$\sigma_{x}=Q_{11}(z) \varepsilon_{x}$

$\tau_{x z}=Q_{55}(z) \gamma_{x z}$

where:

$Q_{11}(z)=\frac{E(z)}{1-v^{2}(z)}, \quad Q_{55}(z)=G_{12}(z)$

The stress resultant forces including axial force $N_{x}$, bending moment $M_{x}$ and shear force $Q_{x}$ are defined as:

$N_{x}=\int_{-h / 2}^{h / 2} \sigma_{x} d z, \quad M_{x}=\int_{-h / 2}^{h / 2} z \sigma_{x} d z, \quad Q_{x}=\int_{-h / 2}^{h / 2} \tau_{x z} d z$

Substituting Eq. 5 into Eq. 7 we have:

$N_{x}=A_{11} \frac{\partial u_{0}}{\partial x}+B_{11} \frac{\partial \Psi}{\partial x}$

$M_{x}=B_{11} \frac{\partial u_{0}}{\partial x}+D_{11} \frac{\partial \Psi}{\partial x}$

$Q_{x}=\kappa A_{55}\left(\frac{\partial w_{0}}{\partial x}+\Psi\right)$

where:

$$
\begin{aligned}
& \left(A_{11}, B_{11}, D_{11}\right)=\int_{-\frac{h}{2}}^{\frac{h}{2}} Q_{11}(z)\left(1, z, z^{2}\right) d z, \quad A_{55}=\int_{-\frac{h}{2}}^{\frac{h}{2}} Q_{55}(z) d z \\
& \left(I_{1}, I_{2}, I_{3}\right)=\int_{-\frac{h}{2}}^{\frac{h}{2}} \rho(z)\left(1, z, z^{2}\right) d z
\end{aligned}
$$

Indicating the stiffness components and inertia terms and $\kappa$ denotes the shear correction factor, taken $5 / 6$ for rectangular cross sections [38].

As shown in Fig. 3 the BNNTRC beam is considered of length $L$, width $b$, thickness $h$, lying on an elastic foundation and subjected to different boundary conditions at edges. $(\mathrm{x}=0$ and $\mathrm{x}=\mathrm{L})$.

The elastic foundation is considered to be Winkler-Pasternak, with reaction force per unit area as: [39]

$P=k_{w} W-k_{s} \frac{\partial^{2} W}{\partial x^{2}}$

Therein $k_{s}$ and $k_{w}$ denote shearing and Winkler layer coefficients of the elastic foundation and $W$ represents the transverse displacement of the beam. Clearly, setting $k_{s}=0$ leads to Winkler type.

\subsection{Equations of motion}

Hamilton's principle is employed to derive the governing differential equations of motion, denoted as:

$\delta \int_{0}^{t}(T-U+V) d t=0$

where $T$ represents the kinetic energy of the beam, $U$ denotes the potential energy consisting of the beam strain

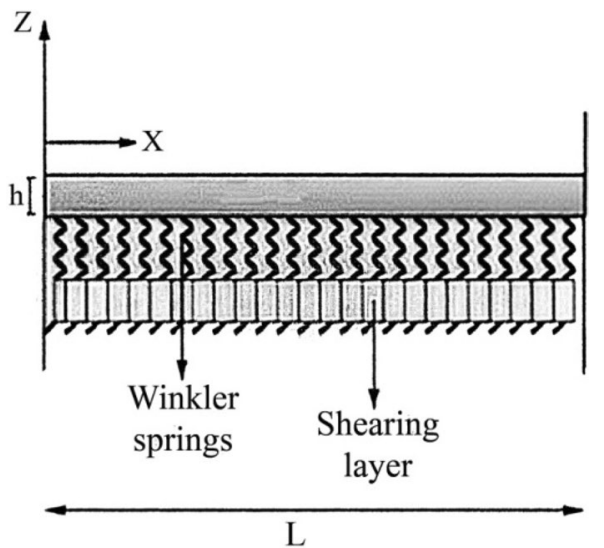

Fig. 3 Geometry of BNNTRC beam 
energy and the potential energy of the elastic foundation, and $V$ expresses the work done by the axial force $\mathrm{N}_{\mathrm{x}}$. Since in free vibration analysis, $N_{x}=0$, we have:

$T=\frac{b}{2} \int_{0}^{l} \int_{-h / 2}^{\mathrm{h} / 2} \rho(z) \dot{u}_{i} \dot{u}_{i} d z d x$

$U=\frac{b}{2} \int_{0}^{1} \int_{-h / 2}^{\mathrm{h} / 2} \sigma_{i j} \varepsilon_{i j} d z d x+\frac{b}{2} \int_{0}^{l}\left(k_{w} W^{2}-k_{s}\left(\frac{\partial W}{\partial x}\right)^{2}\right) d x$

$V=0$

where $\rho(z)$ indicates density of the beam.

Substituting Eq. (12) into Eq. (11) and integrating by parts yields:

$$
\begin{aligned}
\iint_{00}^{t L}[ & -\left(\frac{\partial N_{x}}{\partial x}-I_{1} \frac{\partial^{2} u_{0}}{\partial t^{2}}-I_{2} \frac{\partial^{2} \Psi}{\partial t^{2}}\right) \delta u \\
& -\left(\frac{\partial Q_{x}}{\partial x}-k_{w} w_{0}+k_{s} \frac{\partial^{2} w_{0}}{\partial x^{2}}-I_{1} \frac{\partial^{2} w_{0}}{\partial t^{2}}\right) \delta w \\
& \left.-\left(\frac{\partial M_{x}}{\partial x}-Q_{x}-I_{2} \frac{\partial^{2} u_{0}}{\partial t^{2}}-I_{3} \frac{\partial^{2} \Psi}{\partial t^{2}}\right) \delta \Psi\right] d x d t=0
\end{aligned}
$$

Resulting in:

$$
\begin{aligned}
& \frac{\partial N_{x}}{\partial x}=I_{1} \frac{\partial^{2} u_{0}}{\partial t^{2}}+I_{2} \frac{\partial^{2} \Psi}{\partial t^{2}} \\
& \frac{\partial Q_{x}}{\partial x}-k_{w} w_{0}+k_{s} \frac{\partial^{2} w_{0}}{\partial x^{2}}=I_{1} \frac{\partial^{2} w_{0}}{\partial t^{2}} \\
& \frac{\partial M_{x}}{\partial x}-Q_{x}=I_{2} \frac{\partial^{2} u_{0}}{\partial t^{2}}+I_{3} \frac{\partial^{2} \Psi}{\partial t^{2}}
\end{aligned}
$$

Substituting Eq. (8) into Eq. (14), differential equations of motion are expressed as:

$A_{11} \frac{\partial^{2} u_{0}}{\partial x^{2}}+B_{11} \frac{\partial^{2} \Psi}{\partial x^{2}}=I_{1} \frac{\partial^{2} u_{0}}{\partial t^{2}}+I_{2} \frac{\partial^{2} \Psi}{\partial t^{2}}$
$\kappa A_{55}\left(\frac{\partial^{2} w_{0}}{\partial x^{2}}+\frac{\partial \Psi}{\partial x}\right)-k_{w} w_{0}+k_{s} \frac{\partial^{2} w_{0}}{\partial x^{2}}=I_{1} \frac{\partial^{2} w_{0}}{\partial t^{2}}$
$B_{11} \frac{\partial^{2} u_{0}}{\partial x^{2}}+D_{11} \frac{\partial^{2} \Psi}{\partial x^{2}}-\kappa A_{55}\left(\frac{\partial w_{0}}{\partial x}+\Psi\right)=I_{2} \frac{\partial^{2} u_{0}}{\partial t^{2}}+I_{3} \frac{\partial^{2} \Psi}{\partial t^{2}}$

The BNNTRC beam is subjected to four different boundary conditions, including clamped-clamped (C-C), clamped-free (C-F), clamped-simply supported (C-S) and simply supported-simply supported (S-S), defined as:
C: $\quad u_{0}=w_{0}=\Psi=0$

$S: \quad u_{0}=w_{0}=0$,

$M_{x}=B_{11} \frac{\partial u_{0}}{\partial x}+D_{11} \frac{\partial \Psi}{\partial x}=0$

$F: \quad N_{x}=A_{11} \frac{\partial u_{0}}{\partial x}+B_{11} \frac{\partial \Psi}{\partial x}=0$,

$M_{x}=B_{11} \frac{\partial u_{0}}{\partial x}+D_{11} \frac{\partial \Psi}{\partial x}=0$,

$Q_{x}=\kappa A_{55}\left(\frac{\partial w_{0}}{\partial x}+\Psi\right)=0$

Applying following variable changes, will alter the governing equations to a dimensionless form.

$$
\begin{aligned}
\xi & =\frac{x}{L},(\bar{U}, \bar{W})=\frac{\left(u_{0}, w_{0}\right)}{h}, \\
k_{1} & =\frac{k_{w} L^{2}}{A_{110}}, k_{2}=\frac{k_{s}}{A_{110}}, \eta=\frac{L}{h}, \psi=\Psi\left(\bar{I}_{1}, \bar{I}_{2}, \bar{I}_{3}\right) \\
& =\left(\frac{I_{1}}{I_{10}}, \frac{I_{2}}{I_{10} h}, \frac{I_{3}}{I_{10} h^{2}}\right),\left(a_{11}, b_{11}, d_{11}, a_{55}\right) \\
& =\left(\frac{A_{11}}{A_{110}}, \frac{B_{11}}{A_{110} h^{\prime}}, \frac{D_{11}}{A_{110} h^{2}}, \frac{A_{55}}{A_{110}}\right), \\
\omega & =\Omega L \sqrt{\frac{I_{10}}{A_{110}}}, \tau=\frac{t}{L} \sqrt{\frac{A_{110}}{I_{10}}}
\end{aligned}
$$

where $A_{110}$ and $I_{10}$ represent the same values as $A_{11}$ and $I_{1}$, but of a homogeneous beam comprised of pure matrix material. Substituting Eq. (17) into Eq. (15) leads to the following dimensionless equations of motion:

$a_{11} \frac{\partial^{2} \bar{U}}{\partial \xi^{2}}+b_{11} \frac{\partial^{2} \psi}{\partial \xi^{2}}=\bar{I}_{1} \frac{\partial^{2} \bar{U}}{\partial \tau^{2}}+\bar{I}_{2} \frac{\partial^{2} \psi}{\partial \tau^{2}}$

$\kappa a_{55}\left(\frac{\partial^{2} \bar{W}}{\partial \xi^{2}}+\eta \frac{\partial \psi}{\partial \xi}\right)-k_{1} \bar{W}+k_{2} \frac{\partial^{2} \bar{W}}{\partial \xi^{2}}=\bar{l}_{1} \frac{\partial^{2} \bar{W}}{\partial \tau^{2}}$

$b_{11} \frac{\partial^{2} \bar{U}}{\partial \xi^{2}}+d_{11} \frac{\partial^{2} \psi}{\partial \xi^{2}}-\kappa \eta a_{55}\left(\frac{\partial \bar{W}}{\partial \xi}+\eta \psi\right)=\bar{I}_{2} \frac{\partial^{2} \bar{U}}{\partial \tau^{2}}+\bar{I}_{3} \frac{\partial^{2} \psi}{\partial \tau^{2}}$

To obtain the natural frequencies, the displacements are expressed as following eigenvalue functions:

$\bar{W}(\xi, \tau)=w(\xi) e^{-i \omega \tau}$

$\bar{U}(\xi, \tau)=u(\xi) e^{-i \omega \tau}$

$\psi(\xi, \tau)=\psi(\xi) e^{-i \omega \tau}$

where $i=\sqrt{-1}$ and $\omega$ denotes dimensionless natural frequency. Furthermore $\mathrm{w}(\xi), \mathrm{u}(\xi)$ and $\psi(\xi)$ are the shape modes of transverse, axial and rotational movements, respectively.

Substituting Eq. (19) into Eq. (18) yields: 
$a_{11} \frac{\partial^{2} u}{\partial \xi^{2}}+b_{11} \frac{\partial^{2} \psi}{\partial \xi^{2}}=-\bar{l}_{1} \omega^{2} u-\bar{l}_{2} \omega^{2} \psi$

$\kappa a_{55}\left(\frac{\partial^{2} w}{\partial \xi^{2}}+\eta \frac{\partial \psi}{\partial \xi}\right)-k_{1} w+k_{2} \frac{\partial^{2} w}{\partial \xi^{2}}=-\bar{l}_{1} \omega^{2} w$

$b_{11} \frac{\partial^{2} u}{\partial \xi^{2}}+d_{11} \frac{\partial^{2} \psi}{\partial \xi^{2}}-\kappa \eta a_{55}\left(\frac{\partial W}{\partial \xi}+\eta \psi\right)=-\bar{I}_{2} \omega^{2} u-\bar{I}_{3} \omega^{2} \psi$

Dimensionless boundary conditions are expressed as:

$$
\begin{aligned}
& S: \quad u=w=0 \\
& M_{x}=b_{11} \frac{d u}{d \xi}+d_{11} \frac{d \psi}{d \xi}=0 \\
& C: \quad u=w=\psi=0 \\
& F: \quad N_{x}=a_{11} \frac{d u}{d \xi}+b_{11} \frac{d \psi}{d \xi}=0 \\
& M_{x}=b_{11} \frac{d u}{d \xi}+d_{11} \frac{d \psi}{d \xi}=0 \\
& Q_{x}=\kappa a_{55}\left(\frac{d w}{d \xi}+\psi\right)=0
\end{aligned}
$$

where $\xi=0,1$.

\section{Generalized differential quadrature method (GDQM)}

Generalized differential quadrature method (GDQM) is applied to approximate the nth-order partial derivative of a function $f(x)$ with respect to $x$ at a specific point $x_{i}$, as follows: $[40,41]$

$$
\frac{\partial^{n} f\left(x_{i}\right)}{\partial x^{n}}=\sum_{k=1}^{N} c_{i k}^{(n)} f\left(x_{k}\right), \quad i=1, \ldots, N, n=1, \ldots, N
$$

Therein, $\mathrm{N}$ denotes the sampling points numbers along the beam and $c_{i k}^{(n)}$ indicates the nth-order derivative weighting coefficients.

The weighting coefficients are calculated through Eq. (23a) and (23b), defining the first derivative weighting coefficients as:

$$
\begin{aligned}
& c_{i j}^{(1)}=\frac{M^{(1)}\left(x_{i}\right)}{\left(x_{i}-x_{j}\right) M^{(1)}\left(x_{i}\right)}, \quad i, j=1,2, \ldots, N, \quad i \neq j \\
& c_{i i}^{(1)}=-\sum_{\substack{j=1 \\
i \neq j}}^{N} c_{i j}^{(1)}, \quad i=1,2, \ldots, N \\
& M^{(1)}\left(x_{i}\right)=\prod_{\substack{j=1 \\
i \neq j}}^{N}\left(x_{i}-x_{j}\right), \quad i=1,2, \ldots, N
\end{aligned}
$$

And coefficients of higher-order derivatives as the following recursive relations:

$$
\begin{gathered}
c_{i j}^{(n)}=n\left(c_{i i}^{(n-1)} c_{i j}^{(1)}-\frac{c_{i j}^{(n-1)}}{\left(x_{i}-x_{j}\right)}\right), \quad i, j=1,2, \ldots, \\
N, i \neq j, n=2,3, \ldots, N-1
\end{gathered}
$$

$c_{i i}^{(n)}=-\sum_{j=1}^{N} c_{i j}^{(n)}, \quad i=1,2, \ldots, N, n=1,2, \ldots, N-1$

To achieve the most accurate results, the sampling points are taken as Chebyshev-Gauss-Lobatto distribution, described as:

$x_{i}=\frac{1}{2}\left(1-\cos \left(\frac{i-1}{N-1}\right) \pi\right), i=1,2, \ldots, N$

where $x_{i}$ would be in $[0,1]$ range. Applying GDQ method to Eq. (20) yields:

$$
\begin{aligned}
& a_{11} \sum_{j=1}^{N} c_{i j}^{(2)} u_{j}+b_{11} \sum_{j=1}^{N} c_{i j}^{(2)} \Psi_{j}=-\bar{l}_{1} \omega^{2} u_{i}-\bar{I}_{2} \omega^{2} \Psi_{i} \\
& \kappa a_{55}\left(\sum_{j=1}^{N} c_{i j}^{(2)} w_{j}+\sum_{j=1}^{N} c_{i j}^{(1)} \Psi_{j}\right)-k_{1} w_{i}+k_{2} \sum_{j=1}^{N} c_{i j}^{(2)} w_{j} \\
& =-\bar{l}_{1} \omega^{2} w_{i} \\
& b_{11} \sum_{j=1}^{N} c_{i j}^{(2)} u_{j}+d_{11} \sum_{j=1}^{N} c_{i j}^{(2)} \Psi_{j}-\kappa \eta a_{55}\left(\sum_{j=1}^{N} c_{i j}^{(1)} w_{j}+\eta \Psi_{i}\right) \\
& =-\bar{l}_{2} \omega^{2} u_{i}-\bar{l}_{3} \omega^{2} \Psi_{i}
\end{aligned}
$$

where $c_{i j}^{(1)}$ and $c_{i j}^{(2)}$ indicate the first and second order weighting coefficients, as expressed before.

In the same way applying GDQM to the boundary conditions leads to:

$$
\begin{aligned}
& \text { C: } \quad u=w=\psi=0 \\
& \text { S: } \quad u_{i}=w_{i}=0, \quad M_{x}=b_{11} \sum_{j=1}^{N} c_{i j}^{(1)} u_{j}+d_{11} \sum_{j=1}^{N} c_{i j}^{(1)} \Psi_{j}=0 \\
& F: \quad N_{x}=a_{11} \sum_{j=1}^{N} c_{i j}^{(1)} u_{j}+b_{11} \sum_{j=1}^{N} c_{i j}^{(1)} \Psi_{j}=0, \\
& M_{x}=b_{11} \sum_{j=1}^{N} c_{i j}^{(1)} u_{j}+d_{11} \sum_{j=1}^{N} c_{i j}^{(1)} \Psi_{j}=0, \\
& Q_{x}=\kappa a_{55}\left(\sum_{j=1}^{N} c_{i j}^{(1)} w_{j}+\psi\right)=0
\end{aligned}
$$

where $\mathrm{i}=1$ at $\xi=0, \mathrm{i}=\mathrm{N}$ at $\xi=1$. 


\section{Natural frequencies of the BNNTRC beam}

In order to derive the natural frequencies of the BNNTRC beam, the displacements are considered as:

$$
\left\{\boldsymbol{U}_{\boldsymbol{d}}\right\}=\left\{\left\{\boldsymbol{U}_{\boldsymbol{d}}\right\},\left\{\boldsymbol{W}_{\boldsymbol{d}}\right\},\left\{\boldsymbol{\Psi}_{\boldsymbol{d}}\right\}\right\}^{T},\left\{\boldsymbol{U}_{\boldsymbol{b}}\right\}=\left\{\left\{\boldsymbol{U}_{\boldsymbol{b}}\right\},\left\{\boldsymbol{W}_{\boldsymbol{b}}\right\},\left\{\boldsymbol{\Psi}_{\boldsymbol{b}}\right\}\right\}^{T}
$$

where subscript ' $b$ ' stands for the boundary points and subscript ' $d$ ' denotes the points throughout the domain. Introducing the boundary conditions into Eq. (25) results in the following system of algebraic:

$$
\left[\begin{array}{ll}
{\left[\boldsymbol{T}_{\boldsymbol{b b}}\right]} & {\left[\boldsymbol{T}_{\boldsymbol{b d}}\right.} \\
{\left[\boldsymbol{T}_{\boldsymbol{d b}}\right]} & {\left[\boldsymbol{T}_{\boldsymbol{d d}}\right]}
\end{array}\right]\left\{\begin{array}{l}
\left\{\boldsymbol{U}_{\boldsymbol{b}}\right\} \\
\left\{\boldsymbol{U}_{\boldsymbol{d}}\right\}
\end{array}\right\}=\omega^{2}\left[\begin{array}{ll}
{[\mathbf{0}]} & {[\mathbf{0}]} \\
{[\mathbf{0}]} & \left.\overline{\boldsymbol{I}}_{\boldsymbol{i}}\right]
\end{array}\right]\left\{\begin{array}{l}
\left\{\boldsymbol{U}_{\boldsymbol{b}}\right\} \\
\left\{\boldsymbol{U}_{\boldsymbol{d}}\right\}
\end{array}\right\}
$$

where $\left[\overline{\mathrm{i}}_{\mathrm{i}}\right]$ indicates matrices comprised of dimensionless inertia terms.

Equation (28) leads to following two equations:

$\left[\boldsymbol{T}_{d b}\right]\left\{\boldsymbol{U}_{b}\right\}+\left[\boldsymbol{T}_{d d}\right]\left\{\boldsymbol{U}_{d}\right\}=\left[\bar{I}_{i}\right] \omega^{2}\left\{\boldsymbol{U}_{d}\right\}$

$\left\{\boldsymbol{U}_{\boldsymbol{b}}\right\}=-\left[\boldsymbol{T}_{\boldsymbol{b b}}\right]^{-1}\left[\boldsymbol{T}_{\boldsymbol{b d}}\right]\left\{\boldsymbol{U}_{\boldsymbol{d}}\right\}$

The following eigenvalue problem is yielded by substituting Eq. (29b) in Eq. (29a):

$\left([\boldsymbol{T}]-\omega^{2}\left[\overline{\boldsymbol{I}}_{\boldsymbol{i}}\right]\right)\left\{\boldsymbol{U}_{\boldsymbol{d}}\right\}=\{\boldsymbol{0}\}$

where $[\boldsymbol{T}]=\left[\boldsymbol{T}_{d d}\right]-\left[\boldsymbol{T}_{d b}\right]\left[\boldsymbol{T}_{b b}\right]^{-1}\left[\boldsymbol{T}_{\boldsymbol{b d}}\right]$.

Eventually, solving this problem will result in the natural frequencies of the BNNTRC beam.

\section{Numerical results and discussion}

First and foremost, in order to evaluate the results of this study and to confirm the accuracy of applied method, free vibration of a FGM beam on an elastic foundation is analyzed and the results are then measured against the ones in the literature. The FGM beam properties are presented as to follow the exponential function and the Poisson's ratio is considered to be constant through all beam thickness [42].As shown in Table 1, there is a reliable convergence between the results of the present method and those of the literature. It is also easily observed that taking 9 sampling points is enough to achieve reliable results.

\subsection{Free vibration analysis of composite beam reinforced by aligned BNNTs}

First, the natural frequencies of BNNTRC beam are obtained due to different nanotube distributions and diversity of boundary conditions, and then the results are measured against the similar ones of the CNTRC beam in the literature. The matrix is taken as poly methyl methacrylate (PMMA), and the nanotubes are chosen to be armchair $(10,10)$ SWBNNTs.

The material properties are determined as $\rho_{\mathrm{m}}=1190 \mathrm{~kg} / \mathrm{m}^{3}, \nu_{\mathrm{m}}=0.3, E_{\mathrm{m}}=2.5 \mathrm{Gpa}$ for PMMA and $\rho_{\text {bnnt }}=2270 \mathrm{~kg} / \mathrm{m}^{3}, \nu_{\text {bnnt }}=0.14, \mathrm{E}_{\mathrm{bnnt}}=1.064 \mathrm{Tpa}$ ， $\mathrm{I}_{\mathrm{bnnt}} / \mathrm{d}_{\mathrm{bnnt}}=300$ for nanotubes $[8,43]$. (at room temperature $300 \mathrm{k}$ )

The volume fraction of BNNTs in the matrix is assumed to be $V_{b n n t}^{*}=0.12,0.17,0.28$. It is important to denote that the UD-BNNTRC and FG-BNNTRC beams possess equal mass fractions of BNNT. The foundation models are taken as Pasternak elastic foundation $\left(k_{2}, k_{1}\right)=(0.02,0.1)$, Winkler elastic foundation $\left(k_{2}, k_{1}\right)=(0,0.1)$ and $\left(k_{2}, k_{1}\right)=(0,0)$ represents non-elastic foundation

Tables 2, 3, 4 and 5 illustrate the impacts of different volume fractions of BNNTs $\left(\mathrm{V}_{\mathrm{bnnt}}^{*}\right)$ and the elastic foundation type on the first three dimensionless natural frequencies of BNNTRC beams containing different types of BNNT distribution and subjected to various boundary conditions as (C-C), (C-F), (C-S) and (S-S).

As illustrated, both types of elastic foundations cause an increase in dimensionless natural frequency parameters independent of boundary conditions. It is also observed
Table 1 Accuracy and convergence of the dimensionless frequency parameters of FGM beams $\left(\mathrm{L} / \mathrm{h}=6, E_{2} / E_{1}=0.2\right)$

\begin{tabular}{lllllllll}
\hline$\left(k_{1}, k_{2}\right)$ & BCs & $\mathrm{N}=5$ & $\mathrm{~N}=7$ & $\mathrm{~N}=9$ & $\mathrm{~N}=11$ & $\mathrm{~N}=13$ & Ref. [19] & Ref. [17] \\
\hline$(0,0)$ & $\mathrm{C}-\mathrm{C}$ & 0.8662 & 0.8598 & 0.8600 & 0.8600 & 0.8600 & 0.8600 & 0.8494 \\
$(0.05,0)$ & & 0.9224 & 0.9161 & 0.9163 & 0.9163 & 0.9163 & 0.9159 & \\
$(0.05,0.01)$ & & 1.0276 & 1.0297 & 1.0298 & 1.0298 & 1.0298 & 1.0293 & \\
$(0,0)$ & $\mathrm{C}-\mathrm{F}$ & 0.1486 & 0.1557 & 0.1556 & 0.1556 & 0.1556 & 0.1556 & 0.1547 \\
$(0.05,0)$ & & 0.3496 & 0.3525 & 0.3523 & 0.3523 & 0.3523 & 0.3520 & \\
$(0.05,0.01)$ & & 0.3479 & 0.3372 & 0.3370 & 0.3370 & 0.3370 & 0.3367 & \\
$(0,0)$ & $\mathrm{S}-\mathrm{S}$ & 0.4526 & 0.4600 & 0.4598 & 0.4598 & 0.4598 & 0.4597 & 0.4543 \\
$(0.05,0)$ & & 0.5514 & 0.5573 & 0.5571 & 0.5571 & 0.5571 & 0.5567 & \\
$(0.05,0.01)$ & & 0.7058 & 0.7111 & 0.7108 & 0.7108 & 0.7108 & 0.7103 & \\
\hline
\end{tabular}


Table 2 First three dimensionless natural frequencies of (C-C) BNNTRC beams with aligned BNNTs in the matrix $(L / h=15)$

\begin{tabular}{|c|c|c|c|c|c|c|c|c|c|c|c|c|}
\hline \multirow[t]{2}{*}{$V_{\text {bnnt }}^{*}$} & \multicolumn{3}{|l|}{ UD } & \multicolumn{3}{|l|}{$\mathrm{FG}-\Lambda$} & \multicolumn{3}{|l|}{ FG-X } & \multicolumn{3}{|l|}{$\mathrm{FG}-\diamond$} \\
\hline & 0.12 & 0.17 & 0.28 & 0.12 & 0.17 & 0.28 & 0.12 & 0.17 & 0.28 & 0.12 & 0.17 & 0.28 \\
\hline \multicolumn{13}{|c|}{$\left(k_{1}, k_{2}\right)=(0,0)$} \\
\hline$\omega_{1}$ & 1.4112 & 1.5265 & 1.7233 & 1.3036 & 1.4302 & 1.6673 & 1.5262 & 1.6433 & 1.8828 & 1.2032 & 1.3297 & 1.5593 \\
\hline$\omega_{2}$ & 2.9896 & 3.1794 & 3.5316 & 2.8444 & 3.0610 & 3.5059 & 3.1508 & 3.3514 & 3.8021 & 2.6974 & 2.9201 & 3.3589 \\
\hline$\omega_{3}$ & 4.8034 & 5.0706 & 5.5880 & 4.6296 & 4.9404 & 5.6155 & 5.0045 & 5.2888 & 5.9606 & 4.4461 & 4.7662 & 5.4356 \\
\hline \multicolumn{13}{|c|}{$\left(k_{1}, k_{2}\right)=(0.1,0)$} \\
\hline$\omega_{1}$ & 1.4428 & 1.5546 & 1.7462 & 1.3377 & 1.4601 & 1.691 & 1.5554 & 1.6694 & 1.9039 & 1.2400 & 1.3618 & 1.5846 \\
\hline$\omega_{2}$ & 3.0046 & 3.1930 & 3.5428 & 2.8601 & 3.0751 & 3.5172 & 3.1651 & 3.3642 & 3.8126 & 2.7140 & 2.9348 & 3.3707 \\
\hline$\omega_{3}$ & 4.8127 & 5.0792 & 5.5952 & 4.6393 & 4.9491 & 5.6225 & 5.0135 & 5.2970 & 5.9673 & 4.4562 & 4.7752 & 5.4429 \\
\hline \multicolumn{13}{|c|}{$\left(k_{1}, k_{2}\right)=(0.1,0.02)$} \\
\hline$\omega_{1}$ & 1.5056 & 1.6102 & 1.7916 & 1.4064 & 1.5202 & 1.7386 & 1.6130 & 1.7207 & 1.9452 & 1.3152 & 1.4272 & 1.6361 \\
\hline$\omega_{2}$ & 3.1212 & 3.2985 & 3.6306 & 2.9830 & 3.1849 & 3.6058 & 3.2757 & 3.4644 & 3.8942 & 2.8441 & 3.0503 & 3.4635 \\
\hline$\omega_{3}$ & 4.9754 & 5.2277 & 5.7199 & 4.8074 & 5.1011 & 5.7464 & 5.1701 & 5.4398 & 6.0845 & 4.6310 & 4.9325 & 5.5707 \\
\hline
\end{tabular}

Table 3 First three dimensionless natural frequencies of (C-F) BNNTRC beams with aligned BNNTs in the matrix $(\mathrm{L} / \mathrm{h}=15)$

\begin{tabular}{|c|c|c|c|c|c|c|c|c|c|c|c|c|}
\hline \multirow[t]{2}{*}{$V_{b n n t}^{*}$} & \multicolumn{3}{|l|}{ UD } & \multicolumn{3}{|l|}{$\mathrm{FG}-\Lambda$} & \multicolumn{3}{|l|}{ FG-X } & \multicolumn{3}{|l|}{ FG- $\diamond$} \\
\hline & 0.12 & 0.17 & 0.28 & 0.12 & 0.17 & 0.28 & 0.12 & 0.17 & 0.28 & 0.12 & 0.17 & 0.28 \\
\hline \multicolumn{13}{|c|}{$\left(k_{1}, k_{2}\right)=(0,0)$} \\
\hline$\omega_{1}$ & 0.3274 & 0.3775 & 0.4616 & 0.2778 & 0.321 & 0.3968 & 0.3929 & 0.4534 & 0.561 & 0.2417 & 0.2792 & 0.3439 \\
\hline$\omega_{2}$ & 1.562 & 1.717 & 1.9773 & 1.4097 & 1.5684 & 1.8564 & 1.7338 & 1.8994 & 2.2195 & 1.2809 & 1.4336 & 1.7037 \\
\hline$\omega_{3}$ & 3.4476 & 3.7128 & 4.1802 & 3.2144 & 3.5029 & 4.0626 & 3.7055 & 3.9845 & 4.5668 & 3.0024 & 3.2903 & 3.8311 \\
\hline \multicolumn{13}{|c|}{$\left(k_{1}, k_{2}\right)=(0.1,0)$} \\
\hline$\omega_{1}$ & 0.4442 & 0.4785 & 0.541 & 0.4089 & 0.4354 & 0.4869 & 0.4944 & 0.5405 & 0.628 & 0.3853 & 0.4056 & 0.4449 \\
\hline$\omega_{2}$ & 1.5904 & 1.7419 & 1.9973 & 1.4411 & 1.5956 & 1.8776 & 1.7595 & 1.922 & 2.2374 & 1.3154 & 1.4633 & 1.7268 \\
\hline$\omega_{3}$ & 3.4606 & 3.7244 & 4.1897 & 3.2282 & 3.5151 & 4.0723 & 3.7176 & 3.9953 & 4.5755 & 3.0173 & 3.3033 & 3.8414 \\
\hline \multicolumn{13}{|c|}{$\left(k_{1}, k_{2}\right)=(0.1,0.02)$} \\
\hline$\omega_{1}$ & 0.4353 & 0.472 & 0.5375 & 0.3973 & 0.4258 & 0.4799 & 0.489 & 0.5376 & 0.6277 & 0.3722 & 0.394 & 0.4357 \\
\hline$\omega_{2}$ & 1.6708 & 1.8144 & 2.0575 & 1.5267 & 1.6717 & 1.9389 & 1.8351 & 1.9904 & 2.2934 & 1.407 & 1.5443 & 1.7918 \\
\hline$\omega_{3}$ & 3.591 & 3.8434 & 4.2898 & 3.3633 & 3.6369 & 4.1715 & 3.843 & 4.1099 & 4.6698 & 3.159 & 3.4302 & 3.9442 \\
\hline
\end{tabular}

Table 4 First three dimensionless natural frequencies of (C-S) BNNTRC beams with aligned BNNTs in the matrix $(\mathrm{L} / \mathrm{h}=15)$

\begin{tabular}{|c|c|c|c|c|c|c|c|c|c|c|c|c|}
\hline \multirow[t]{2}{*}{$V_{b n n t}^{*}$} & \multicolumn{3}{|l|}{ UD } & \multicolumn{3}{|l|}{$\mathrm{FG}-\Lambda$} & \multicolumn{3}{|l|}{ FG-X } & \multicolumn{3}{|l|}{ FG- $\diamond$} \\
\hline & 0.12 & 0.17 & 0.28 & 0.12 & 0.17 & 0.28 & 0.12 & 0.17 & 0.28 & 0.12 & 0.17 & 0.28 \\
\hline \multicolumn{13}{|c|}{$\left(k_{1}, k_{2}\right)=(0,0)$} \\
\hline$\omega_{1}$ & 1.1384 & 1.2559 & 1.4505 & 1.048 & 1.1715 & 1.3942 & 1.2705 & 1.3943 & 1.6292 & 0.9228 & 1.0368 & 1.237 \\
\hline$\omega_{2}$ & 2.8399 & 3.0542 & 3.4313 & 2.6635 & 2.9006 & 3.3603 & 3.0452 & 3.2679 & 3.7366 & 2.4743 & 2.7123 & 3.1573 \\
\hline$\omega_{3}$ & 4.7229 & 5.007 & 5.5391 & 4.52 & 4.8501 & 5.5386 & 4.9521 & 5.2482 & 5.9281 & 4.2988 & 4.6389 & 5.3205 \\
\hline \multicolumn{13}{|c|}{$\left(k_{1}, k_{2}\right)=(0.1,0)$} \\
\hline$\omega_{1}$ & 1.1773 & 1.2899 & 1.4777 & 1.0901 & 1.2078 & 1.4225 & 1.3055 & 1.425 & 1.6535 & 0.9703 & 1.0777 & 1.2688 \\
\hline$\omega_{2}$ & 2.8557 & 3.0683 & 3.4429 & 2.6802 & 2.9155 & 3.3721 & 3.0599 & 3.2811 & 3.7473 & 2.4924 & 2.7282 & 3.1699 \\
\hline$\omega_{3}$ & 4.7324 & 5.0157 & 5.5463 & 4.5299 & 4.8589 & 5.5458 & 4.9612 & 5.2564 & 5.9348 & 4.3092 & 4.6482 & 5.328 \\
\hline \multicolumn{13}{|c|}{$\left(k_{1}, k_{2}\right)=(0.1,0.02)$} \\
\hline$\omega_{1}$ & 1.2538 & 1.3567 & 1.5313 & 1.1732 & 1.2797 & 1.4787 & 1.3737 & 1.4849 & 1.701 & 1.0644 & 1.1592 & 1.3327 \\
\hline$\omega_{2}$ & 2.9772 & 3.1774 & 3.5328 & 2.8095 & 3.03 & 3.4637 & 3.1737 & 3.3834 & 3.8302 & 2.6316 & 2.8507 & 3.2674 \\
\hline$\omega_{3}$ & 4.8979 & 5.1662 & 5.6722 & 4.7019 & 5.0137 & 5.6713 & 5.1196 & 5.4005 & 6.0528 & 4.4898 & 4.8098 & 5.4586 \\
\hline
\end{tabular}


Table 5 First three dimensionless natural frequencies of (S-S) BNNTRC beams with aligned BNNTs in the matrix $(\mathrm{L} / \mathrm{h}=15)$

\begin{tabular}{|c|c|c|c|c|c|c|c|c|c|c|c|c|}
\hline \multirow[t]{2}{*}{$V_{b n n t}^{*}$} & \multicolumn{3}{|l|}{ UD } & \multicolumn{3}{|l|}{$\mathrm{FG}-\Lambda$} & \multicolumn{3}{|l|}{$F G-X$} & \multicolumn{3}{|l|}{$F G-\varnothing$} \\
\hline & 0.12 & 0.17 & 0.28 & 0.12 & 0.17 & 0.28 & 0.12 & 0.17 & 0.28 & 0.12 & 0.17 & 0.28 \\
\hline \multicolumn{13}{|c|}{$\left(k_{1}, k_{2}\right)=(0,0)$} \\
\hline$\omega_{1}$ & 0.8625 & 0.9802 & 1.1749 & 0.8444 & 0.9661 & 1.1826 & 1.0103 & 1.1449 & 1.3854 & 0.6546 & 0.7504 & 0.9156 \\
\hline$\omega_{2}$ & 2.663 & 2.9042 & 3.3087 & 2.4247 & 2.6818 & 3.1526 & 2.9174 & 3.1648 & 3.6527 & 2.2214 & 2.473 & 2.9219 \\
\hline$\omega_{3}$ & 4.6391 & 4.9427 & 5.4918 & 4.4184 & 4.7695 & 5.4739 & 4.9003 & 5.2098 & 5.8992 & 4.1383 & 4.5016 & 5.1984 \\
\hline \multicolumn{13}{|c|}{$\left(k_{1}, k_{2}\right)=(0.1,0)$} \\
\hline$\omega_{1}$ & 0.9132 & 1.0233 & 1.2083 & 0.8961 & 1.0098 & 1.2158 & 1.0539 & 1.1821 & 1.4138 & 0.72 & 0.8059 & 0.9581 \\
\hline$\omega_{2}$ & 2.6798 & 2.919 & 3.3207 & 2.4431 & 2.6978 & 3.1651 & 2.9327 & 3.1784 & 3.6636 & 2.2415 & 2.4903 & 2.9355 \\
\hline$\omega_{3}$ & 4.6488 & 4.9514 & 5.499 & 4.4285 & 4.7785 & 5.4812 & 4.9095 & 5.2181 & 5.906 & 4.1491 & 4.5112 & 5.206 \\
\hline \multicolumn{13}{|c|}{$\left(k_{1}, k_{2}\right)=(0.1,0.02)$} \\
\hline$\omega_{1}$ & 1.0058 & 1.1036 & 1.2717 & 0.9903 & 1.0912 & 1.2788 & 1.1351 & 1.2522 & 1.4684 & 0.8343 & 0.9056 & 1.0368 \\
\hline$\omega_{2}$ & 2.8091 & 3.0337 & 3.414 & 2.5835 & 2.8209 & 3.2625 & 3.0515 & 3.2841 & 3.7485 & 2.3942 & 2.6235 & 3.0404 \\
\hline$\omega_{3}$ & 4.8175 & 5.1041 & 5.6261 & 4.6047 & 4.9362 & 5.6084 & 5.0696 & 5.3633 & 6.0245 & 4.3367 & 4.6779 & 5.3398 \\
\hline
\end{tabular}

that the Pasternak type causes higher fundamental frequencies compared to Winkler type, except for C-F boundary condition. It can also be indicated that raising BNNT volume fraction $\left(\mathrm{V}_{\mathrm{bnnt}}^{*}\right)$ causes a growth in the dimensionless fundamental frequency parameters.

Furthermore, it is noticeable that the highest and the lowest frequency parameters are obtained using $\mathrm{FG}-\mathrm{X}$ and FG- $\triangle$ BNNTRC distributions, respectively, leading to a conclusion that symmetric functionally graded distributions of BNNTs have a substantial impact on rising or declining the frequency parameters. Comparing the results on the basis of boundary conditions demonstrates that the highest frequencies are observed in the $C-C$ beams, followed by C-S, S-S and C-F beams respectively.

Figure 4 illustrates the impact of nanotubes distribution type through the beam thickness on the first fundamental frequency parameter versus slenderness ratio for $\mathrm{C}-\mathrm{C}$ beams, where the beam is resting on Pasternak foundation $\left(k_{2}, k_{1}\right)=(0.02,0.1)$ and $V_{b n n t}^{*}=0.28$. As the figure demonstrates, the highest natural frequencies are observed in FG-X BNNTRC beams, compared to the beams containing other BNNTs distributions. It is also observed that, while the slenderness ratio increases the frequency parameter experiences a decrease for all distribution types. Moreover, the influence of BNNTs
Fig. 4 Effect of BNNTs distribution type on the first frequency parameter of BNNTRC beam with aligned layout in the matrix

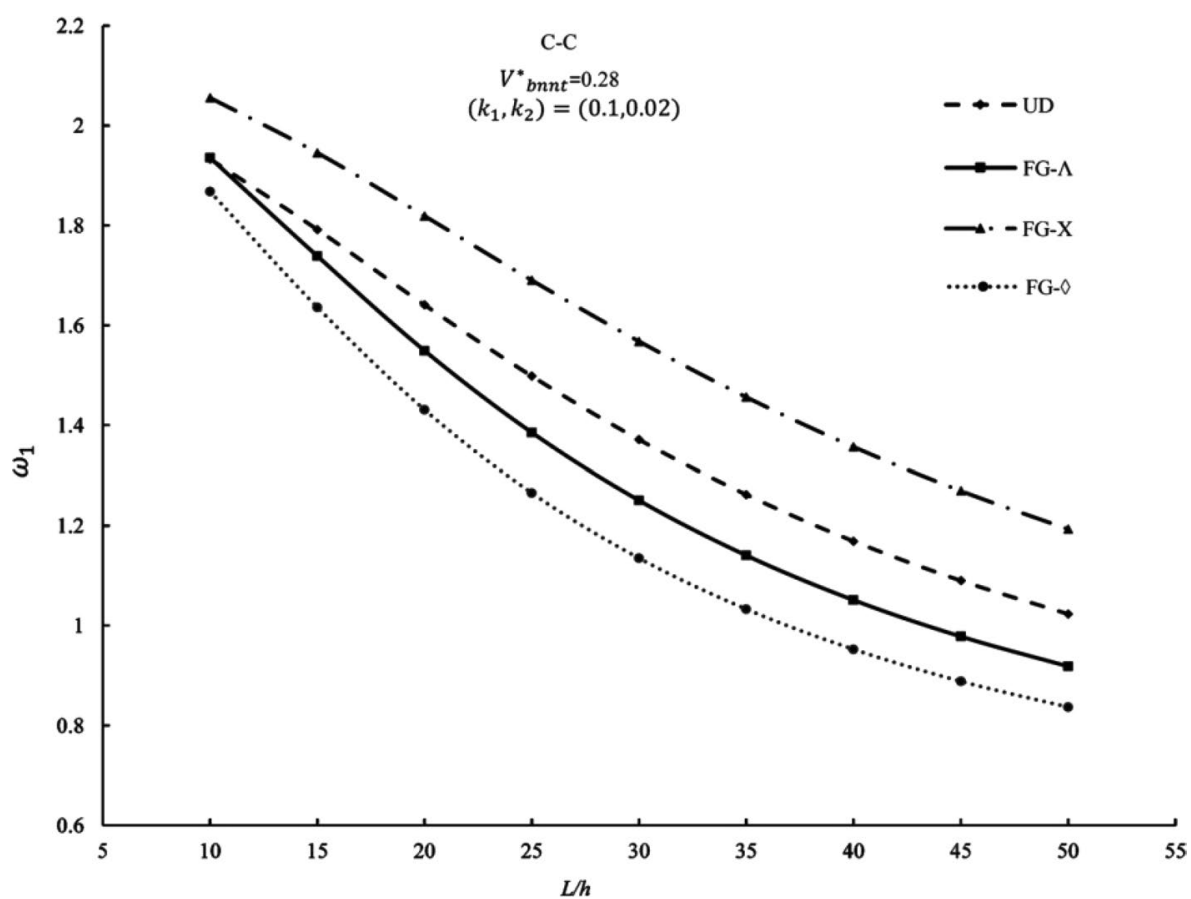

SN Applied Sciences 
Fig. 5 The effect of BNNTs volume fraction on the first frequency parameter of BNNTRC beams with aligned layout in the matrix
Fig. 6 The effect of boundary conditions on the first frequency parameter of BNNTRC beams with aligned layout in the matrix
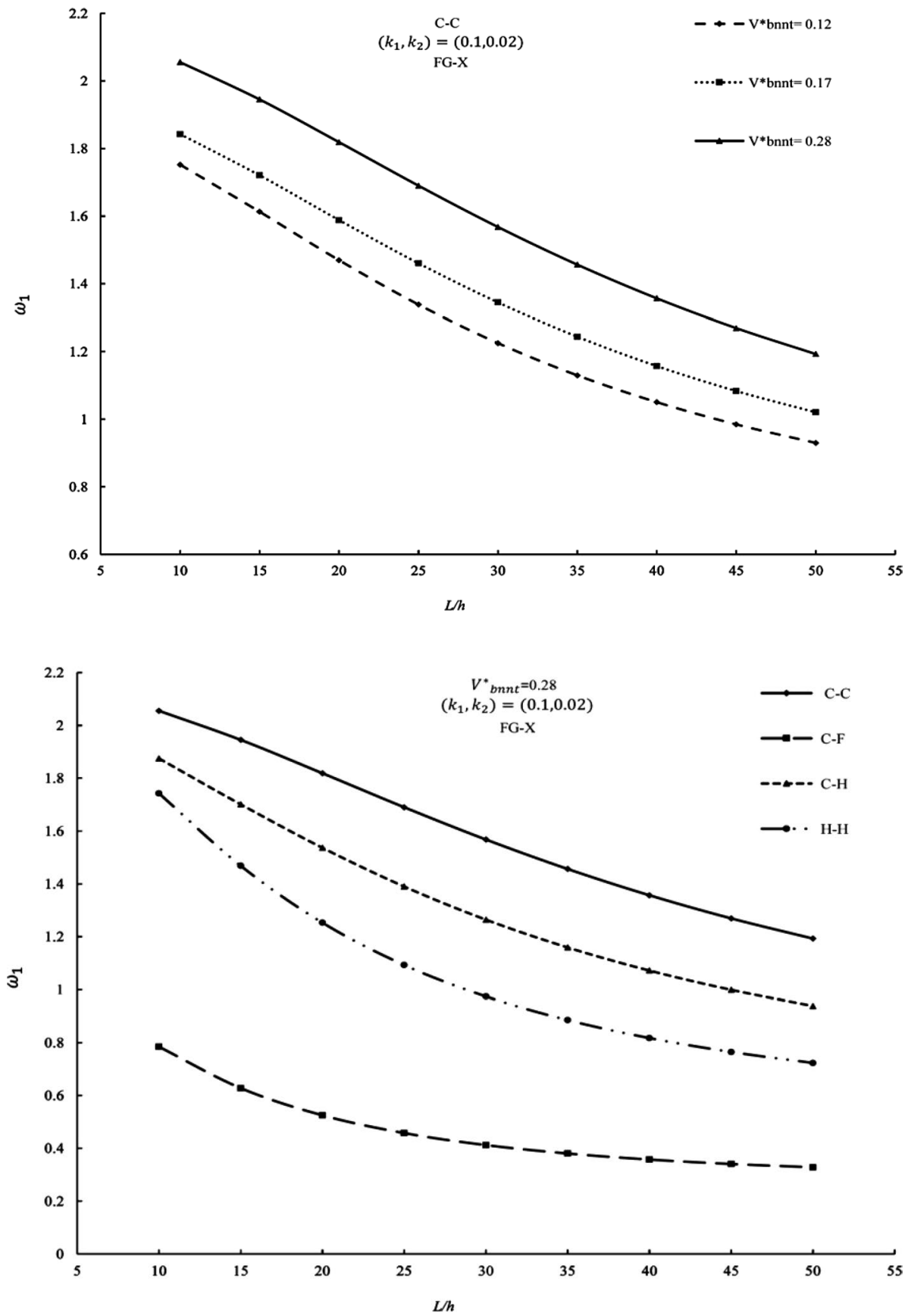

volume fraction on frequency of the BNNTRC beams is presented in Fig. 5, showing that the frequencies of the BNNTRC beams increase with any rise in the BNNTs volume fraction $\left(\mathrm{V}_{\mathrm{bnnt}}^{*}\right)$.

Figure 6 indicates the impact of boundary conditions on the first frequency parameter. As shown, the highest frequencies are obtained under C-C condition followed by $\mathrm{C}-\mathrm{S}, \mathrm{S}-\mathrm{S}$ and $\mathrm{C}-\mathrm{F}$ conditions respectively.

Therefore, the effect of elastic foundation on the first frequency parameter is indicated for $\mathrm{C}-\mathrm{C}$ and $\mathrm{C}-\mathrm{F}$ conditions in Fig. 7, showing that under both boundary conditions, the frequency parameters of the beams on elastic foundation are higher than those of the beams without any elastic foundation. In C-C condition Pasternak foundation results in higher frequency compared to Winkler type, while in cantilever beam a different behavior is observed; the frequency of $\mathrm{C}-\mathrm{F}$ beam on Winkler foundation is slightly higher than its frequency on Pasternak foundation. 
Fig. 7 The effect of foundation parameters on the first frequency parameter of BNNTRC beams with aligned layout in the matrix subjected to $\mathrm{C}-\mathrm{C}$ and $\mathrm{C}-\mathrm{F}$ boundary conditions

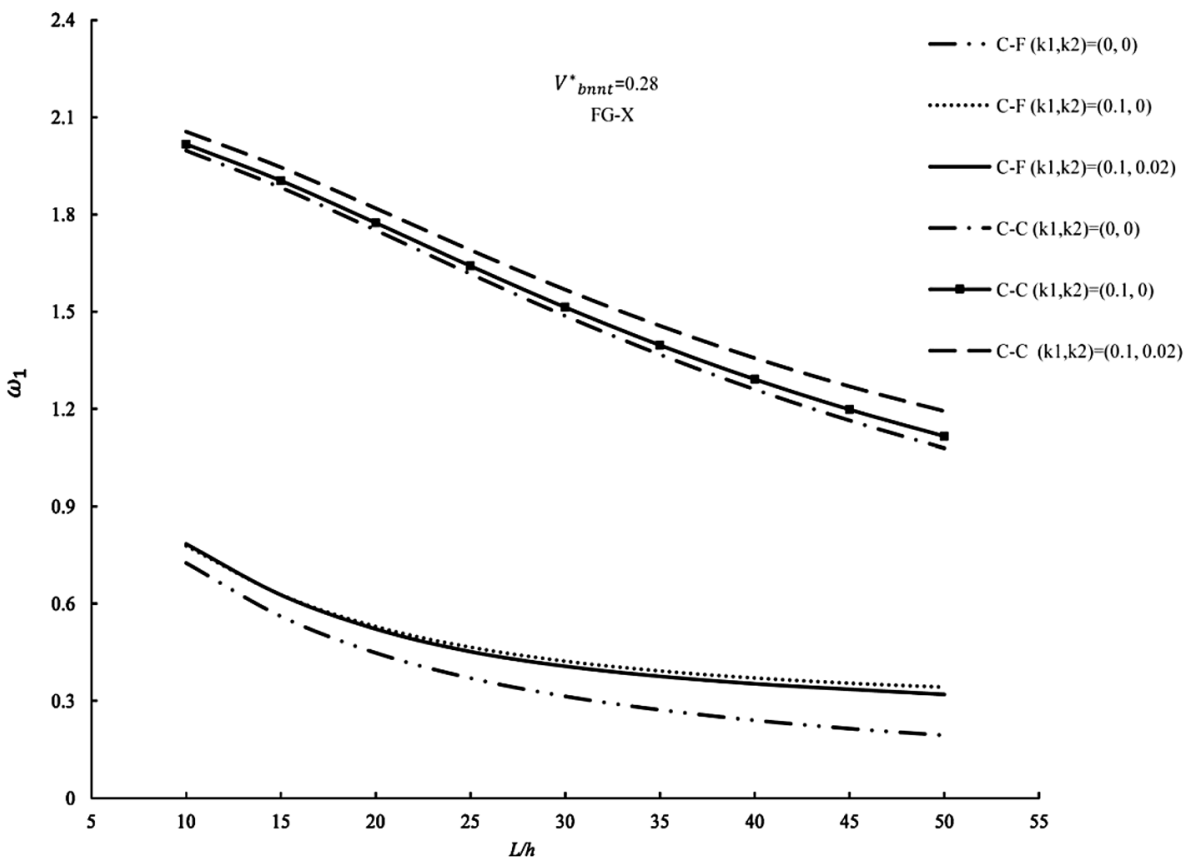

Table 6 Comparing the first dimensionless frequency parameters of (C-C) BNNTRC beam with (C-C) CNTRC beam [18] $(\mathrm{L} / \mathrm{h}=15)$

\begin{tabular}{|c|c|c|c|c|c|c|c|c|c|}
\hline \multirow[t]{3}{*}{$V^{*}$} & \multirow{2}{*}{\multicolumn{2}{|c|}{$\left(k_{1}, k_{2}\right)=(0,0)$}} & \multirow{3}{*}{$\begin{array}{l}\text { Difference } \\
\text { percent }\end{array}$} & \multirow{2}{*}{\multicolumn{2}{|c|}{$\left(k_{1}, k_{2}\right)=(0.1,0)$}} & \multirow{3}{*}{$\begin{array}{l}\text { Difference } \\
\text { percent }\end{array}$} & \multirow{2}{*}{\multicolumn{2}{|c|}{ (k) }} & \multirow{3}{*}{$\begin{array}{l}\text { Difference } \\
\text { percent }\end{array}$} \\
\hline & & & & & & & & & \\
\hline & CNTRC [18] & BNNTRC & & CNTRC [18] & BNNTRC & & CNTRC [18] & BNNTRC & \\
\hline \multicolumn{10}{|l|}{0.12} \\
\hline UD & 1.5085 & 1.4112 & 6.45 & 1.5406 & 1.4428 & 6.34 & 1.6038 & 1.5056 & 6.12 \\
\hline $\mathrm{FG}-\Lambda$ & 1.4068 & 1.3036 & 7.33 & 1.4412 & 1.3377 & 7.18 & 1.5096 & 1.4064 & 6.83 \\
\hline FG- $\diamond$ & 1.318 & 1.2032 & 8.71 & 1.3546 & 1.24 & 8.46 & 1.4282 & 1.3152 & 7.91 \\
\hline FG-X & 1.6 & 1.5262 & 4.61 & 1.6303 & 1.5554 & 4.59 & 1.6895 & 1.613 & 4.52 \\
\hline \multicolumn{10}{|l|}{0.17} \\
\hline UD & 1.9144 & 1.5265 & 20.26 & 1.9396 & 1.5546 & 19.84 & 1.9901 & 1.6102 & 19.08 \\
\hline $\mathrm{FG}-\Lambda$ & 1.7721 & 1.4302 & 19.29 & 1.7993 & 1.4601 & 18.85 & 1.8546 & 1.5202 & 18.03 \\
\hline FG- $\diamond$ & 1.65 & 1.3297 & 19.41 & 1.6791 & 1.3618 & 18.89 & 1.7393 & 1.4272 & 17.94 \\
\hline FG-X & 2.0498 & 1.6433 & 19.83 & 2.0799 & 1.6694 & 19.73 & 2.12 & 1.7207 & 18.83 \\
\hline \multicolumn{10}{|l|}{0.28} \\
\hline UD & 2.1618 & 1.7233 & 20.28 & 2.1837 & 1.7462 & 20.03 & 2.2274 & 1.7916 & 19.56 \\
\hline $\mathrm{FG}-\Lambda$ & 2.0504 & 1.6673 & 18.68 & 2.0735 & 1.691 & 18.44 & 2.1202 & 1.7386 & 17.99 \\
\hline FG- $\diamond$ & 1.9284 & 1.5593 & 19.14 & 1.953 & 1.5846 & 18.86 & 2.0032 & 1.6361 & 18.32 \\
\hline FG-X & 2.3169 & 1.8828 & 18.73 & 2.3374 & 1.9039 & 18.54 & 2.3779 & 1.9452 & 18.19 \\
\hline
\end{tabular}

Tables 6, 7, 8 and 9 compare the frequencies of BNNTRC beams with those of CNTRC beams in the literature [18], of the same matrix, nanotube volume fractions and also under the same boundary conditions. It should be also mentioned that in this comparison, the nanotubes are assumed to have an aligned layout in the matrix and they are also considered to be uniformly or functionally graded distributed through the thickness direction.
It could be generally concluded that the first dimensionless frequency parameters of BNNTRC beams are lower than those of CNNTRC beams. The difference between frequency parameters of two reinforced beams varies due to nanotubes distribution and volume fraction in the matrix. Moreover, it also depends on different boundary conditions and elastic foundation type. However the effect of foundation coefficient is not very noticeable, the 
Table 7 Comparing the first dimensionless frequency parameters of (C-F) BNNTRC beam with (C-F) CNTRC beam [18] (L/h $=15)$

\begin{tabular}{|c|c|c|c|c|c|c|c|c|c|}
\hline \multirow[t]{3}{*}{$V^{*}$} & \multicolumn{2}{|l|}{$\omega_{1}$} & \multirow{3}{*}{$\begin{array}{l}\text { Difference } \\
\text { percent }\end{array}$} & \multicolumn{2}{|l|}{$\omega_{1}$} & \multirow{3}{*}{$\begin{array}{l}\text { Difference } \\
\text { percent }\end{array}$} & \multicolumn{2}{|l|}{$\omega_{1}$} & \multirow{3}{*}{$\begin{array}{l}\text { Difference } \\
\text { percent }\end{array}$} \\
\hline & \multicolumn{2}{|l|}{$\left(k_{1}, k_{2}\right)=(0,0)$} & & \multicolumn{2}{|c|}{$\left(k_{1}, k_{2}\right)=(0.1,0)$} & & \multicolumn{2}{|c|}{$\left(k_{1}, k_{2}\right)=(0.1,0.02)$} & \\
\hline & CNTRC [18] & BNNTRC & & CNTRC [18] & BNNTRC & & CNTRC [18] & BNNTRC & \\
\hline \multicolumn{10}{|l|}{0.12} \\
\hline UD & 0.3764 & 0.3274 & 13.01 & 0.4893 & 0.4442 & 9.21 & 0.4825 & 0.4353 & 9.78 \\
\hline $\mathrm{FG}-\Lambda$ & 0.3193 & 0.2778 & 12.99 & 0.4469 & 0.4089 & 8.50 & 0.4368 & 0.3973 & 9.04 \\
\hline$F G-\diamond$ & 0.2809 & 0.2417 & 13.95 & 0.4203 & 0.3853 & 8.32 & 0.4083 & 0.3722 & 8.84 \\
\hline FG-X & 0.4416 & 0.3929 & 11.02 & 0.5411 & 0.4944 & 8.63 & 0.538 & 0.489 & 9.10 \\
\hline \multicolumn{10}{|l|}{0.17} \\
\hline UD & 0.4587 & 0.3775 & 17.70 & 0.5544 & 0.4785 & 13.69 & 0.5471 & 0.472 & 13.72 \\
\hline $\mathrm{FG}-\Lambda$ & 0.3866 & 0.321 & 16.96 & 0.4963 & 0.4354 & 12.27 & 0.486 & 0.4258 & 12.38 \\
\hline$F G-\diamond$ & 0.3394 & 0.2792 & 17.73 & 0.4606 & 0.4056 & 11.94 & 0.4483 & 0.394 & 12.11 \\
\hline FG-X & 0.5413 & 0.453 & 16.31 & 0.6245 & 0.541 & 13.37 & 0.6203 & 0.538 & 13.26 \\
\hline \multicolumn{10}{|l|}{0.28} \\
\hline UD & 0.5612 & 0.4616 & 17.74 & 0.6404 & 0.541 & 15.52 & 0.6359 & 0.5375 & 15.47 \\
\hline $\mathrm{FG}-\Lambda$ & 0.4761 & 0.3968 & 16.65 & 0.5673 & 0.4869 & 14.17 & 0.5595 & 0.4799 & 14.22 \\
\hline$F G-\diamond$ & 0.4197 & 0.3439 & 18.06 & 0.5209 & 0.4449 & 14.59 & 0.511 & 0.4357 & 14.73 \\
\hline FG-X & 0.6586 & 0.561 & 14.81 & 0.7273 & 0.628 & 13.65 & 0.7256 & 0.628 & 13.45 \\
\hline
\end{tabular}

Table 8 Comparing the first dimensionless frequency parameters of (C-S) BNNTRC beam with (C-S) CNTRC beam [18] (L/h=15)

\begin{tabular}{|c|c|c|c|c|c|c|c|c|c|}
\hline \multirow[t]{3}{*}{$V^{*}$} & \multirow{2}{*}{\multicolumn{2}{|c|}{$\left(k_{1}, k_{2}\right)=(0,0)$}} & \multirow{3}{*}{$\begin{array}{l}\text { Difference } \\
\text { percent }\end{array}$} & \multirow{2}{*}{\multicolumn{2}{|c|}{$\left(k_{1}, k_{2}\right)=(0.1,0)$}} & \multirow{3}{*}{$\begin{array}{l}\text { Difference } \\
\text { percent }\end{array}$} & \multicolumn{2}{|c|}{$\underline{\omega_{1}}$} & \multirow{3}{*}{$\begin{array}{l}\text { Difference } \\
\text { percent }\end{array}$} \\
\hline & & & & & & & \multicolumn{2}{|c|}{$\left(k_{1}, k_{2}\right)=(0.1,0.02)$} & \\
\hline & CNTRC [18] & BNNTRC & & CNTRC [18] & BNNTRC & & CNTRC [18] & BNNTRC & \\
\hline \multicolumn{10}{|l|}{0.12} \\
\hline UD & 1.2444 & 1.1384 & 8.51 & 1.2831 & 1.1773 & 8.24 & 1.3586 & 1.2538 & 7.71 \\
\hline $\mathrm{FG}-\Lambda$ & 1.1529 & 1.048 & 9.09 & 1.1945 & 1.0901 & 8.74 & 1.2762 & 1.1732 & 8.07 \\
\hline$F G-\diamond$ & 1.0331 & 0.9228 & 10.67 & 1.0794 & 0.9703 & 10.10 & 1.1705 & 1.0644 & 9.06 \\
\hline FG-X & 1.3577 & 1.2705 & 6.42 & 1.3932 & 1.3055 & 6.29 & 1.4622 & 1.3737 & 6.05 \\
\hline \multicolumn{10}{|l|}{0.17} \\
\hline UD & 1.5602 & 1.2559 & 19.50 & 1.591 & 1.2899 & 18.92 & 1.6523 & 1.3567 & 17.89 \\
\hline $\mathrm{FG}-\Lambda$ & 1.4344 & 1.1715 & 18.32 & 1.4678 & 1.2078 & 17.71 & 1.5349 & 1.2797 & 16.62 \\
\hline$F G-\diamond$ & 1.2769 & 1.0368 & 18.80 & 1.3134 & 1.0777 & 17.94 & 1.3902 & 1.1549 & 16.92 \\
\hline FG-X & 1.7188 & 1.3943 & 18.87 & 1.7467 & 1.425 & 18.41 & 1.802 & 1.4849 & 17.59 \\
\hline \multicolumn{10}{|l|}{0.28} \\
\hline UD & 1.804 & 1.4505 & 19.59 & 1.8302 & 1.4777 & 19.26 & 1.8822 & 1.5313 & 18.64 \\
\hline $\mathrm{FG}-\Lambda$ & 1.6933 & 1.3942 & 17.66 & 1.7212 & 1.4225 & 17.35 & 1.7772 & 1.4787 & 16.79 \\
\hline$F G-\diamond$ & 1.5229 & 1.237 & 18.77 & 1.5538 & 1.2688 & 18.34 & 1.6166 & 1.3327 & 17.56 \\
\hline FG-X & 1.9813 & 1.6292 & 17.77 & 2.0052 & 1.6535 & 17.53 & 2.0523 & 1.701 & 17.11 \\
\hline
\end{tabular}

non-elastic foundation causes the largest difference percent between frequency parameters of two reinforced beams.

Comparing the results on the basis of nanotubes volume fraction reveals that the difference percent between
CNTRC and BNNTRC frequencies increases as the volume fraction increases, but for $\mathrm{V}_{\mathrm{bnnt}}^{*}=0.17$ and 0.28 this difference is almost as the same. Focusing on boundary conditions, it could be inferred that the difference percent between fundamental frequencies of BNNTRC and 
Table 9 Comparing the first dimensionless frequency parameters of (S-S) BNNTRC beam with (S-S) CNTRC beam [18] (L/h=15)

\begin{tabular}{|c|c|c|c|c|c|c|c|c|c|}
\hline \multirow[t]{3}{*}{$V^{*}$} & \multicolumn{2}{|l|}{$\omega_{1}$} & \multirow{3}{*}{$\begin{array}{l}\text { Difference } \\
\text { percent }\end{array}$} & \multicolumn{2}{|l|}{$\omega_{1}$} & \multirow{3}{*}{$\begin{array}{l}\text { Difference } \\
\text { percent }\end{array}$} & \multicolumn{2}{|l|}{$\omega_{1}$} & \multirow{3}{*}{$\begin{array}{l}\text { Differ- } \\
\text { ence } \\
\text { percent }\end{array}$} \\
\hline & \multicolumn{2}{|l|}{$\left(k_{1}, k_{2}\right)=(0,0)$} & & \multicolumn{2}{|c|}{$\left(k_{1}, k_{2}\right)=(0.1,0)$} & & \multicolumn{2}{|c|}{$\left(k_{1}, k_{2}\right)=(0.1,0.02)$} & \\
\hline & CNTRC [18] & BNNTRC & & CNTRC [18] & BNNTRC & & CNTRC [18] & BNNTRC & \\
\hline \multicolumn{10}{|l|}{0.12} \\
\hline UD & 0.9753 & 0.8625 & 11.56 & 1.0241 & 0.9132 & 10.82 & 1.1144 & 1.0058 & 9.74 \\
\hline $\mathrm{FG}-\Lambda$ & 0.9453 & 0.8444 & 10.67 & 0.9957 & 0.8961 & 10.00 & 1.0883 & 0.9903 & 9.00 \\
\hline $\mathrm{FG}-\diamond$ & 0.7527 & 0.6546 & 13.03 & 0.815 & 0.72 & 11.65 & 0.9258 & 0.8343 & 9.88 \\
\hline FG-X & 1.115 & 1.0103 & 9.39 & 1.1581 & 1.0539 & 8.99 & 1.2386 & 1.1351 & 8.35 \\
\hline \multicolumn{10}{|l|}{0.17} \\
\hline UD & 1.1999 & 0.9802 & 18.31 & 1.2396 & 1.0233 & 17.44 & 1.3145 & 1.1036 & 16.04 \\
\hline $\mathrm{FG}-\Lambda$ & 1.1609 & 0.9661 & 16.78 & 1.2019 & 1.0098 & 15.98 & 1.279 & 1.0912 & 14.68 \\
\hline$F G-\diamond$ & 0.9155 & 0.7504 & 18.03 & 0.967 & 0.8059 & 16.66 & 1.0612 & 0.9056 & 14.66 \\
\hline FG-X & 1.383 & 1.1449 & 17.21 & 1.4176 & 1.1821 & 16.61 & 1.4836 & 1.2522 & 15.59 \\
\hline \multicolumn{10}{|l|}{0.28} \\
\hline UD & 1.4401 & 1.1749 & 18.41 & 1.4728 & 1.2038 & 18.26 & 1.5352 & 1.2717 & 17.16 \\
\hline $\mathrm{FG}-\Lambda$ & 1.4027 & 1.1826 & 15.69 & 1.4362 & 1.2158 & 15.34 & 1.5002 & 1.2788 & 14.75 \\
\hline FG- $\diamond$ & 1.1202 & 0.9156 & 18.26 & 1.1619 & 0.9581 & 17.54 & 1.24 & 1.0368 & 16.38 \\
\hline FG-X & 1.6493 & 1.3854 & 16.00 & 1.6779 & 1.4138 & 15.74 & 1.733 & 1.4684 & 15.26 \\
\hline
\end{tabular}

Table 10 First three dimensionless natural frequencies of (C-C) BNNTRC beams with randomly oriented BNNTs in the matrix $(\mathrm{L} / \mathrm{h}=15)$

\begin{tabular}{|c|c|c|c|c|c|c|c|c|c|c|c|c|}
\hline \multirow{2}{*}{$V_{b n n t}^{*}$} & \multicolumn{3}{|l|}{ UD } & \multicolumn{3}{|l|}{$\mathrm{FG}-\Lambda$} & \multicolumn{3}{|l|}{ FG-X } & \multicolumn{3}{|l|}{ FG- $\diamond$} \\
\hline & 0.12 & 0.17 & 0.28 & 0.12 & 0.17 & 0.28 & 0.12 & 0.17 & 0.28 & 0.12 & 0.17 & 0.28 \\
\hline \multicolumn{13}{|c|}{$\left(k_{1}, k_{2}\right)=(0,0)$} \\
\hline$\omega_{1}$ & 1.5384 & 1.7973 & 2.2495 & 1.3128 & 1.5235 & 1.8992 & 1.8593 & 2.194 & 2.8046 & 1.1382 & 1.3161 & 1.627 \\
\hline$\omega_{2}$ & 4.0368 & 4.7148 & 5.8996 & 3.4817 & 4.0426 & 5.0439 & 4.7916 & 5.648 & 7.2084 & 3.0464 & 3.5247 & 4.3624 \\
\hline$\omega_{3}$ & 7.4629 & 8.7139 & 10.901 & 6.5091 & 7.5617 & 9.4432 & 8.7013 & 10.246 & 13.057 & 5.7541 & 6.6621 & 8.2563 \\
\hline \multicolumn{13}{|c|}{$\left(k_{1}, k_{2}\right)=(0.1,0)$} \\
\hline$\omega_{1}$ & 1.5673 & 1.8211 & 2.2671 & 1.3465 & 1.5515 & 1.92 & 1.8834 & 2.2136 & 2.8187 & 1.177 & 1.3484 & 1.6512 \\
\hline$\omega_{2}$ & 4.0478 & 4.7239 & 5.9063 & 3.4945 & 4.0531 & 5.0516 & 4.8009 & 5.6555 & 7.2139 & 3.061 & 3.5368 & 4.3714 \\
\hline$\omega_{3}$ & 7.4688 & 8.7187 & 10.904 & 6.5159 & 7.5672 & 9.4473 & 8.7064 & 10.25 & 13.06 & 5.7617 & 6.6684 & 8.261 \\
\hline \multicolumn{13}{|c|}{$\left(k_{1}, k_{2}\right)=(0.1,0.02)$} \\
\hline$\omega_{1}$ & 1.6343 & 1.8769 & 2.3086 & 1.4243 & 1.617 & 1.9692 & 1.9388 & 2.2591 & 2.8517 & 1.2657 & 1.4237 & 1.7085 \\
\hline$\omega_{2}$ & 4.1437 & 4.8031 & 5.9648 & 3.6058 & 4.1458 & 5.1205 & 4.881 & 5.721 & 7.2611 & 3.1887 & 3.6437 & 4.4516 \\
\hline$\omega_{3}$ & 7.5782 & 8.809 & 10.971 & 6.6412 & 7.6713 & 9.5243 & 8.7994 & 10.326 & 13.115 & 5.905 & 6.7879 & 8.3503 \\
\hline
\end{tabular}

CNTRC beam has its lowest amount under C-C condition and on the contrary the highest difference percent is observed in $\mathrm{C}-\mathrm{F}$ beams.

\subsection{Free vibration of composite beams reinforced by randomly oriented BNNTs}

The properties of randomly oriented BNNT-reinforced beams are estimated through Eqs. (38)-(44) in the "Appendix". The distribution of BNNTs through beam thickness is considered as the same for composite beam reinforced by aligned BNNTs.
As inferred from Tables 10, 11, 12 and 13 the fundamental frequencies of the composite beam reinforced by randomly oriented BNNTs, almost goes through the same procedure as the frequencies of composite beam reinforced by aligned BNNTs, due to various boundary conditions, elastic foundation type, slenderness ratio, various BNNTs volume fraction and distribution type. As indicated in Tables 10, 11, 12 and 13 the fundamental frequency of randomly oriented BNNTRC beam increases with increasing the BNNT volume fraction $\left(\mathrm{V}_{\mathrm{bnnt}}^{*}\right)$ under any boundary condition. 
Table 11 First three dimensionless natural frequencies of $(\mathrm{C}-\mathrm{F})$ BNNTRC beams with randomly oriented BNNTs in the matrix $(\mathrm{L} / \mathrm{h}=15)$

\begin{tabular}{|c|c|c|c|c|c|c|c|c|c|c|c|c|}
\hline \multirow[t]{2}{*}{$V_{\text {bnnt }}^{*}$} & \multicolumn{3}{|l|}{ UD } & \multicolumn{3}{|l|}{$\mathrm{FG}-\Lambda$} & \multicolumn{3}{|l|}{ FG-X } & \multicolumn{3}{|l|}{ FG- $\diamond$} \\
\hline & 0.12 & 0.17 & 0.28 & 0.12 & 0.17 & 0.28 & 0.12 & 0.17 & 0.28 & 0.12 & 0.17 & 0.28 \\
\hline \multicolumn{13}{|c|}{$\left(k_{1}, k_{2}\right)=(0,0)$} \\
\hline$\omega_{1}$ & 0.2511 & 0.2934 & 0.3673 & 0.212 & 0.246 & 0.3064 & 0.2902 & 0.3645 & 0.4666 & 0.1826 & 0.211 & 0.2607 \\
\hline$\omega_{2}$ & 1.5301 & 1.7878 & 2.2378 & 1.2993 & 1.5076 & 1.8791 & 1.7506 & 2.196 & 2.8081 & 1.125 & 1.3007 & 1.6077 \\
\hline$\omega_{3}$ & 4.1448 & 4.8415 & 6.0589 & 3.5504 & 4.1216 & 5.1411 & 4.6698 & 5.8484 & 7.4681 & 3.1019 & 3.5884 & 4.4404 \\
\hline \multicolumn{13}{|c|}{$\left(k_{1}, k_{2}\right)=(0.1,0)$} \\
\hline$\omega_{1}$ & 0.3912 & 0.4154 & 0.4632 & 0.3674 & 0.3833 & 0.4165 & 0.4048 & 0.4684 & 0.5453 & 0.3512 & 0.362 & 0.3841 \\
\hline$\omega_{2}$ & 1.559 & 1.8116 & 2.2554 & 1.3331 & 1.5357 & 1.8999 & 1.773 & 2.2154 & 2.8221 & 1.164 & 1.3332 & 1.632 \\
\hline$\omega_{3}$ & 4.1554 & 4.8503 & 6.0653 & 3.5627 & 4.1317 & 5.1486 & 4.6782 & 5.8557 & 7.4733 & 3.1161 & 3.6002 & 4.4491 \\
\hline \multicolumn{13}{|c|}{$\left(k_{1}, k_{2}\right)=(0.1,0.02)$} \\
\hline$\omega_{1}$ & 0.3742 & 0.3994 & 0.4495 & 0.35 & 0.3665 & 0.4015 & 0.3898 & 0.4539 & 0.5336 & 0.3341 & 0.3449 & 0.3682 \\
\hline$\omega_{2}$ & 1.6329 & 1.8733 & 2.3015 & 1.418 & 1.6074 & 1.954 & 1.8314 & 2.2665 & 2.8593 & 1.26 & 1.415 & 1.6946 \\
\hline$\omega_{3}$ & 4.2523 & 4.9304 & 6.1245 & 3.6742 & 4.2245 & 5.2175 & 4.7551 & 5.9224 & 7.5215 & 3.2438 & 3.7071 & 4.5293 \\
\hline
\end{tabular}

Table 12 First three dimensionless natural frequencies of (C-S) BNNTRC beams with randomly oriented BNNTs in the matrix $(\mathrm{L} / \mathrm{h}=15)$

\begin{tabular}{|c|c|c|c|c|c|c|c|c|c|c|c|c|}
\hline \multirow[t]{2}{*}{$V_{b n n t}^{*}$} & \multicolumn{3}{|l|}{ UD } & \multicolumn{3}{|l|}{ FG- $\Lambda$} & \multicolumn{3}{|l|}{ FG-X } & \multicolumn{3}{|l|}{ FG- $\diamond$} \\
\hline & 0.12 & 0.17 & 0.28 & 0.12 & 0.17 & 0.28 & 0.12 & 0.17 & 0.28 & 0.12 & 0.17 & 0.28 \\
\hline \multicolumn{13}{|c|}{$\left(k_{1}, k_{2}\right)=(0,0)$} \\
\hline$\omega_{1}$ & 1.0793 & 1.2611 & 1.5785 & 0.9423 & 1.0961 & 1.3719 & 1.3149 & 1.5522 & 1.9855 & 0.7919 & 0.9155 & 1.1313 \\
\hline$\omega_{2}$ & 3.367 & 3.9332 & 4.9224 & 2.9013 & 3.3699 & 4.2074 & 4.0407 & 4.7658 & 6.0878 & 2.5105 & 2.9037 & 3.5916 \\
\hline$\omega_{3}$ & 6.6773 & 7.7982 & 9.757 & 5.776 & 6.7085 & 8.3748 & 7.88 & 9.2846 & 11.843 & 5.0734 & 5.8715 & 7.2711 \\
\hline \multicolumn{13}{|c|}{$\left(k_{1}, k_{2}\right)=(0.1,0)$} \\
\hline$\omega_{1}$ & 1.1201 & 1.2948 & 1.6035 & 0.9888 & 1.1348 & 1.4005 & 1.3486 & 1.5798 & 2.0054 & 0.8467 & 0.9615 & 1.1659 \\
\hline$\omega_{2}$ & 3.3802 & 3.9441 & 4.9304 & 2.9165 & 3.3825 & 4.2166 & 4.0517 & 4.7748 & 6.0943 & 2.5282 & 2.9183 & 3.6026 \\
\hline$\omega_{3}$ & 6.6839 & 7.8036 & 9.761 & 5.7835 & 6.7147 & 8.3793 & 7.8856 & 9.2892 & 11.846 & 5.082 & 5.8787 & 7.2764 \\
\hline \multicolumn{13}{|c|}{$\left(k_{1}, k_{2}\right)=(0.1,0.02)$} \\
\hline$\omega_{1}$ & 1.2076 & 1.3683 & 1.6588 & 1.0865 & 1.2176 & 1.4632 & 1.4218 & 1.6403 & 2.0496 & 0.9598 & 1.0588 & 1.2412 \\
\hline$\omega_{2}$ & 3.4891 & 4.0341 & 4.997 & 3.0416 & 3.4868 & 4.2941 & 4.1423 & 4.8489 & 6.1478 & 2.6731 & 3.04 & 3.6941 \\
\hline$\omega_{3}$ & 6.8033 & 7.9021 & 9.8336 & 5.9195 & 6.8276 & 8.4628 & 7.9865 & 9.3716 & 11.906 & 5.2392 & 6.0099 & 7.3745 \\
\hline
\end{tabular}

Table 13 First three dimensionless natural frequency of (S-S) BNNTRC beams with randomly oriented BNNTs in the matrix $(\mathrm{L} / \mathrm{h}=15)$

\begin{tabular}{|c|c|c|c|c|c|c|c|c|c|c|c|c|}
\hline \multirow{2}{*}{$V_{b n n t}^{*}$} & \multicolumn{3}{|l|}{ UD } & \multicolumn{3}{|l|}{$\mathrm{FG}-\Lambda$} & \multicolumn{3}{|l|}{ FG-X } & \multicolumn{3}{|l|}{$\mathrm{FG}-\diamond$} \\
\hline & 0.12 & 0.17 & 0.28 & 0.12 & 0.17 & 0.28 & 0.12 & 0.17 & 0.28 & 0.12 & 0.17 & 0.28 \\
\hline \multicolumn{13}{|c|}{$\left(k_{1}, k_{2}\right)=(0,0)$} \\
\hline$\omega_{1}$ & 0.701 & 0.8192 & 1.0255 & 0.6841 & 0.8025 & 1.0183 & 0.8598 & 1.0155 & 1.2996 & 0.5109 & 0.5905 & 0.7295 \\
\hline$\omega_{2}$ & 2.7274 & 3.1866 & 3.9885 & 2.3093 & 2.6789 & 3.3375 & 3.3076 & 3.9035 & 4.9905 & 2.011 & 2.3252 & 2.8746 \\
\hline$\omega_{3}$ & 5.8944 & 6.8852 & 8.6162 & 5.1309 & 5.9651 & 7.4596 & 7.0396 & 8.2999 & 10.597 & 4.4182 & 5.1113 & 6.3254 \\
\hline \multicolumn{13}{|c|}{$\left(k_{1}, k_{2}\right)=(0.1,0)$} \\
\hline$\omega_{1}$ & 0.7624 & 0.8703 & 1.0635 & 0.7469 & 0.8546 & 1.0566 & 0.9106 & 1.0571 & 1.3298 & 0.5924 & 0.6596 & 0.7821 \\
\hline$\omega_{2}$ & 2.7437 & 3.2 & 3.9984 & 2.3282 & 2.6946 & 3.3491 & 3.321 & 3.9144 & 4.9984 & 2.033 & 2.3435 & 2.8883 \\
\hline$\omega_{3}$ & 5.9019 & 6.8913 & 8.6207 & 5.1393 & 5.9721 & 7.4647 & 7.0459 & 8.305 & 10.6 & 4.4281 & 5.1196 & 6.3315 \\
\hline \multicolumn{13}{|c|}{$\left(k_{1}, k_{2}\right)=(0.1,0.02)$} \\
\hline$\omega_{1}$ & 0.871 & 0.9632 & 1.1349 & 0.8576 & 0.9493 & 1.1286 & 1.0032 & 1.1348 & 1.3875 & 0.7268 & 0.7781 & 0.8766 \\
\hline$\omega_{2}$ & 2.8689 & 3.3038 & 4.0754 & 2.4728 & 2.8155 & 3.4393 & 3.4252 & 3.9998 & 5.0601 & 2.1991 & 2.4835 & 2.994 \\
\hline$\omega_{3}$ & 6.033 & 6.9995 & 8.7006 & 5.2873 & 6.0949 & 7.5555 & 7.1562 & 8.3951 & 10.665 & 4.601 & 5.2641 & 6.4398 \\
\hline
\end{tabular}


Fig. 8 Variations of the first frequency parameter of FG-X BNNTRC beam subjected to different boundary conditions due to two different BNNTs orientation versus slenderness ratio

Fig. 9 Variations of the first frequency parameter of $\mathrm{C}-\mathrm{C}$ BNNTRC beam due to different nanotubes distribution type versus slenderness ratio for aligned and randomly oriented BNNTRC beams
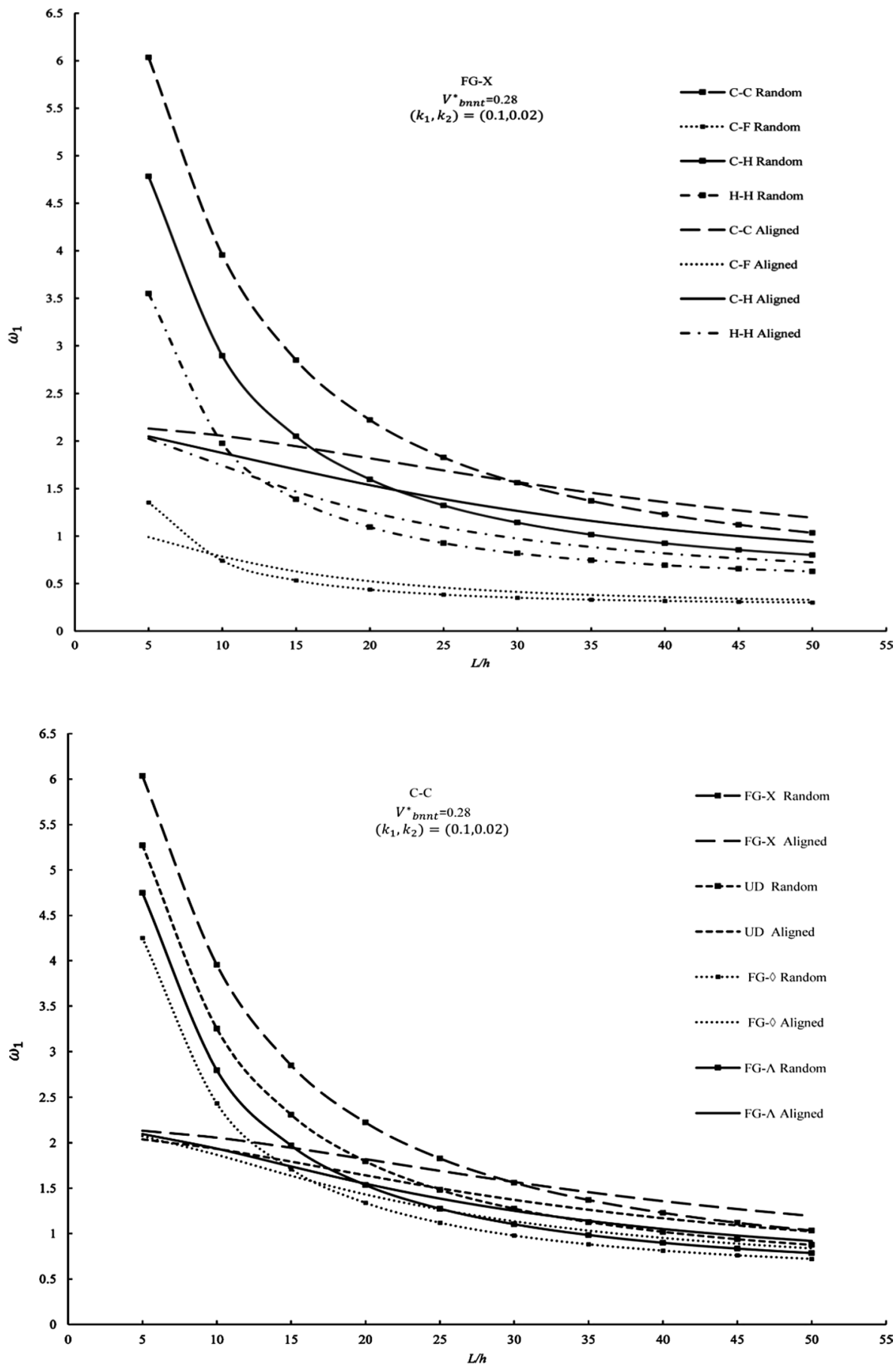

Focusing on the effect of nanotubes distribution type, it is indicated that beams with FG-X nanotubes distribution results in higher natural frequencies, followed by UD, FG-^ and FG- $\diamond$ respectively. It is also represented that resting on an elastic foundation causes higher frequencies, in a way that frequency parameters obtained for Pasternak foundation is higher than the ones for Winkler foundation, except for C-F beams (as also observed in aligned BNNTRC beams). The effect of boundary conditions on frequency parameters is such that $\mathrm{C}-\mathrm{C}$ boundary condition causes the highest frequency and it gets lower in C-S, S-S and C-F conditions respectively.

Figures 8 and 9 illustrate the difference between frequencies obtained for randomly oriented BNNTRC beam and the composite beam reinforced by aligned BNNTs. As displayed in Figs. 8 and 9, the fundamental frequency reduces as the slenderness ratio goes up, regardless of BNNTs orientation in the matrix.

As illustrated in these figures, in small slenderness ratios, the frequencies of randomly oriented BNNTRC 
beam are much higher than frequencies of aligned BNNTRC beam, but as slenderness ratio increases, this difference gets smaller, where in a specific slenderness ratio this difference gets zero and for higher slenderness ratios a different procedure is observed; the randomly oriented BNNTRC beam shows lower frequencies than aligned BNNTRC beam. It is also inferred that the slenderness ratio where equal frequencies are obtained for composite beams with two different orientations of BNNTs, gets different values due to various boundary conditions and nanotubes distribution types.

As shown in Fig. 8, in C-C beams the frequency of two beams equals in higher slenderness ratios followed by C-S, S-S and C-F respectively. Hence, the following figure is represented for C-C beams as a sample. Figure 9 represents the effect of nanotubes distribution type on the difference between frequencies of aligned and randomly oriented BNNTRC beams under C-C boundary conditions, as shown in this figure, the slenderness ratio that this difference equals zero, experiences its smallest amount for $F G-\diamond$, increasing in $F G-\Lambda, U D$ and $F G-X$ distribution type respectively.

\section{Conclusions}

In this paper, free vibration of BNNT reinforced Timoshenko beams on an elastic foundation was studied. The orientation of nanotubes in the matrix was considered to be unidirectional or randomly oriented and a micromechanical model was employed to approximate the mechanical properties of BNNTRC beams. The governing equations were derived through the Hamilton's principle and the natural frequencies of BNNTRC beams were obtained using generalized differential quadrature method. The beam was considered to be lying on both elastic and nonelastic foundations, under various boundary conditions. The obtained results led to the conclusion that the volume fraction of BNNTs and their orientation and distribution type as well as foundation stiffness and the boundary conditions have substantial impacts on the natural frequencies of the BNNTRC beams. That is to say, focusing on orientation of BNNTs revealed that in small slenderness ratios the natural frequencies of randomly oriented BNNTRC beams are higher than aligned BNNTRC beams but for long beams the procedure gets contrariwise. The effect of other contributing factors on natural frequencies of both randomly oriented and aligned BNNTRC beams is the same and is described as following. Beams with more volume fraction of BNNTs show higher natural frequencies. The beams with FG-X distribution showed higher fundamental frequency compared to other distribution types, subsequently UD, FG-^ and FG- $\diamond$ nanotube distributions demonstrate high natural frequencies, leading to a conclusion that symmetric functionally graded distributions of BNNTs have a substantial impact on rising or declining the frequency parameters. Other than that, beams on elastic foundation have higher frequencies than beams on non-elastic foundations. It is also worth mentioning that elastic foundations of larger elastic factors yield higher natural frequencies. The impact of boundary conditions is such that $\mathrm{C}-\mathrm{C}$ beam has the highest natural frequency followed by C-S, S-S and C-F respectively. Comparing the results with the ones of aligned CNTRC beams in the literature revealed that CNTRC beams have higher natural frequency than aligned BNNTRC beams and this difference increases with raising nanotubes volume fraction. It is also noteworthy that, while the slenderness ratio increases the frequency parameter experiences a decrease for all distribution types.

\section{Compliance with ethical standards}

Conflict of interest On behalf of all authors, the corresponding author states that there is no conflict of interest.

\section{Appendix}

\section{BNNTRC beams reinforced by unidirectional distributed BNNTs}

When the assumption is based on reinforcing the composite beam by BNNTs which are unidirectionally distributed among the beam, the elastic properties are then calculated using Eqs. (31-37) based on unidirectional discontinuous fiber reinforced composites [44].

$$
\begin{aligned}
& E_{11}=\frac{1+2\left(I_{b n n t} / d_{b n n t}\right) \eta_{l} V_{b n n t}}{1-\eta_{l} V_{b n n t}} E_{m} \\
& \eta_{I}=\frac{\left(E_{b n n t} / E_{m}\right)-1}{\left(E_{b n n t} / E_{m}\right)+2\left(I_{b n n t} / d_{b n n t}\right)} \\
& E_{22}=\frac{1+2 \eta_{T} V_{b n n t}}{1-\eta_{T} V_{b n n t}} E_{m} \\
& \eta_{T}=\frac{\left(E_{b n n t} / E_{m}\right)-1}{\left(E_{b n n t} / E_{m}\right)+2} \\
& G_{12}=\frac{1+\eta_{G} V_{b n n t}}{1-\eta_{G} V_{b n n t}} G_{m} \\
& \eta_{G}=\frac{\left(G_{b n n t} / G_{m}\right)-1}{\left(G_{b n n t} / G_{m}\right)+1}
\end{aligned}
$$


where $E_{11}$ and $E_{22}$ denote longitudinal and transverse modulus, $G_{b n n t}$ and $G_{m}$ are shear modulus of BNNTs and the isotropic matrix, respectively. $E_{m}$ is the Young's modulus of the pure isotropic matrix, and $E_{b n n t}$ denotes the Young's modulus of BNNTs. $\nu_{\text {bnnt }},{ }_{\text {bnnt }}$ and $d_{b n n t}$ represent Poisson's ratio, average length and diameter of BNNTs. Poisson's ratio and mass density are calculated from:

$v_{12}=V_{b n n t} \nu^{b n n t}+V_{m} v^{m}$

$v_{21}=\frac{E_{22}}{E_{11}} v_{12}$

$\rho_{c}=V_{b n n t} \rho^{b n n t}+V_{m} \rho^{m}$

where $v_{12}$ and $v_{21}$ are major Poisson's ratio and minor Poisson's ratio and $V_{b n n t}$ and $V_{m}$ refer to volume fractions of BNNTs and the matrix, which are related through:

$V_{m}+V_{b n n t}=1$

\section{BNNTRC beams reinforced by randomly oriented BNNTs}

For randomly oriented distribution, the young's modulus and shear modulus are calculated from: [44]

$E_{c}=\frac{3}{8} E_{11}+\frac{5}{8} E_{22}$

$G_{c}=\frac{1}{8} E_{11}+\frac{1}{4} E_{22}$

where $E_{11}$ and $E_{22}$ are the longitudinal and transverse tensile moduli described as:

$E_{11}=\frac{1+2\left(I_{b n n t} / d_{b n n t}\right) \eta_{l} V_{b n n t}}{1-\eta_{l} V_{b n n t}} E_{m}$

$\eta_{l}=\frac{\left(E_{\text {bnnt }} / E_{m}\right)-1}{\left(E_{\text {bnnt }} / E_{m}\right)+2\left(I_{\text {bnnt }} / d_{\text {bnnt }}\right)}$

$E_{22}=\frac{1+2 \eta_{T} V_{b n n t}}{1-\eta_{T} V_{b n n t}} E_{m}$

$\eta_{T}=\frac{\left(E_{\text {bnnt }} / E_{m}\right)-1}{\left(E_{\text {bnnt }} / E_{m}\right)+2}$

where $G_{b n n t}$ and $G_{m}$ are shear modulus of BNNTs and the pure isotropic matrix, respectively. $E_{m}$ and $E_{b n n t}$ are the Young's modulus of the pure isotropic matrix and BNNTs, and $v_{\text {bnnt }}$ represents Poisson's ratio. The proportion of average BNNT length $I_{b n n t}$ to BNNT diameter $d_{b n n t}$ is called nanotube's aspect ratio which is the same for both unidirectional and randomly oriented BNNT reinforced composites. The nanotube's aspect ratio has a significant effect on the longitudinal modulus $\left(E_{11}\right)$, but the transverse modulus $\left(E_{22}\right)$ is not affected by it.

For randomly oriented BNNTRC beams the Poisson's ratio is the same in all directions as follows:

$\nu_{c}=\frac{E_{c}}{2 G_{c}}-1$

And mass density is defined as:

$\rho_{c}=V_{\text {bnnt }} \rho^{\text {bnnt }}+V_{m} \rho^{m}$

$V_{b n n t}$ and $V_{m}$ are the volume fractions of BNNTs and the matrix, which are the same as volume fractions of BNNTs and the matrix in unidirectionally reinforced BNNTRCs, and follows the same relation as:

$V_{m}+V_{b n n t}=1$

\section{References}

1. Li Ch, Chou T (2006) Static and dynamic properties of singlewalled boron nitride nanotubes. J Nanosci Nanotechnol 6:54-60

2. Cohen ML, Zettl A (2010) The physics of boron nitride nanotubes. Phys Today 63:34-39. https://doi.org/10.1063/1.3518210

3. Zhi C, Bando $Y$, Tang $C$ et al (2010) Boron nitride nanotubes. Mater Sci Eng, R 70:92-111. https://doi.org/10.1016/j. mser.2010.06.004

4. Wang Sh, Wang G (2018) Mechanical properties and thermal shock resistance analysis of $\mathrm{BNNT} / \mathrm{Si}_{3} \mathrm{~N}_{4}$ composites. Appl Compos Mater 25:415-423. https://doi.org/10.1007/s1044 3-017-9627-3

5. Santosh M, Prabal K, Sood AK (2009) Elastic properties of boron nitride nanotubes and their comparison with carbon nanotubes. J Nanosci Nanotechnol 9:5425-5430. https://doi.org/10.1166/ jnn.2009.1197

6. Hurst J (2014) Nanotube superfiber materials. National Aeronautics and Space Administrations (NASA), Glenn Research Center, Clevend, OH, USA

7. Zheng F, Zhou G et al (2005) Structural characterizations and electronic properties of boron nitride nanotube crystalline bundles. J Chem Phys 123:124716. https://doi.org/10.1063/1.20350 97

8. Zhang Z, Gou W, Dai Y (2009) Stability and electronic properties of small boron nitride nanotubes. J Appl Phys 105:084705. https ://doi.org/10.1063/1.3115446

9. Yakobson Bl, Avouris P (2001) Mechanical properties of carbon nanotubes. Top Appl Phys 80:287-327. https://doi. org/10.1007/3-540-39947-X_12

10. Garel J, Leven I, Zhi C et al (2012) Ultrahigh torsional stiffness and strength of boron nitride nanotubes. Nano Lett 12:63476352. https://doi.org/10.1021/nl303601d

11. Trivedi S, Sharma SC, Harsha SP (2013) Vibration analysis of boron nitride nanotube reinforced composite based nanomechanical resonator for fixed-fixed configuration. Int J Eng Res Technol 2:5

12. Liew KM, Lei ZX, Zhang LW (2015) Mechanical analysis of functionally graded carbon nanotube reinforced composites: a 
review. Compos Struct 120:90-97. https://doi.org/10.1016/j. compstruct.2014.09.041

13. Liew KM, Pan Z, Zhang LW (2020) The recent progress of functionally graded CNT reinforced composites and structures. Sci China-Phys Mech Astron 63:234601. https://doi.org/10.1007/ s11433-019-1457-2

14. Yas MH, Tahouneh V (2012) 3-D free vibration analysis of thick functionally graded annular plates on Pasternak elastic foundation via differential quadrature method (DQM). Acta Mech 223:43. https://doi.org/10.1007/s00707-011-0543-6

15. Zhang LW (2017) On the study of the effect of in-plane forces on the frequency parameters of CNT-reinforced composite skew plates. Compos Struct 160:824-837. https://doi.org/10.1016/j. compstruct.2016.10.116

16. Zhang LW (2017) An element-free based IMLS-Ritz method for buckling analysis of nanocomposite plates of polygonal planform. Eng Anal Bound Elem 77:10-25. https://doi.org/10.1016/j. enganabound.2017.01.004

17. Ke LL, Yang J, Kitipornchai S et al (2013) Flexural vibration and elastic buckling of a cracked Timoshenko beam made of functionally graded materials. Mech Adv Mater Struct 16:488-502. https://doi.org/10.1080/15376490902781175

18. Yas MH, Samadi N (2012) Free vibrations and buckling analysis of carbon nanotube-reinforced composite Timoshenko beams on elastic foundation. Int J Press Vessels Pip 98:119-128. https ://doi.org/10.1016/j.ijpvp.2012.07.012

19. Yan T, Kitipornchai S, Yang J et al (2011) Dynamic behaviour of edge-cracked shear deformable functionally graded beams on an elastic foundation under a moving load. Compos Struct 93:2992-3001. https://doi.org/10.1016/j.compstruct .2011 .05 .003

20. Liew KM, Pan Z, Zhang LW (2019) An overview of layerwise theories for composite laminates and structures: development, numerical implementation and application. Compos Struct 216:240-259. https://doi.org/10.1016/j.compstruct.2019.02.074

21. Zhang LW, Selim BA (2017) Vibration analysis of CNT-reinforced thick laminated composite plates based on Reddy's higherorder shear deformation theory. Compos Struct 160:689-705. https://doi.org/10.1016/j.compstruct.2016.10.102

22. Shooshtari A, Rafiee $M$ (2011) Nonlinear forced vibration analysis of clamped functionally graded beams. Acta Mech 221:23. https ://doi.org/10.1007/s00707-011-0491-1

23. Cong Z, Lee $S$ (2018) Study of mechanical behavior of BNNT-reinforced aluminum composites using molecular dynamics simulations. Compos Struct 194:80-86. https://doi.org/10.1016/j. compstruct.2018.03.103

24. Thomas B, Roy T (2016) Vibration analysis of functionally graded carbon nanotube-reinforced composite shell structures. Acta Mech 227:581. https://doi.org/10.1007/s00707-015-1479-z

25. Zhi C, Bando Y, Wang WL et al (2008) Mechanical and thermal properties of polymethyl methacrylate-BN nanotube composites. J Nanomater 2008:1-5. https://doi.org/10.1155/2008/64203 6

26. Li L, Chen Y, Stachurski ZH (2013) Boron nitride nanotube reinforced polyurethane composites. Prog Nat Sci: Mater Int 23:170173. https://doi.org/10.1016/j.pnsc.2013.03.004

27. Trivedia S, Sharmaa SC, Harshaa SP (2014) Evaluations of young's modulus of boron nitride nanotube reinforced nano-composites. Procedia Mater Sci 6:1899-1905. https://doi.org/10.1016/j. mspro.2014.07.222

28. Zhang LW, Xiao LN (2017) Mechanical behavior of laminated CNT-reinforced composite skew plates subjected to dynamic loading. Compos Struct 122:219-230. https://doi.org/10.1016/j. compositesb.2017.03.041
29. Zhang LW, Song ZG, Qiao P, Liew KM (2017) Modeling of dynamic responses of CNT-reinforced composite cylindrical shells under impact loads. Comput Methods Appl Mech Eng 313:889-903. https://doi.org/10.1016/j.cma.2016.10.020

30. Pan Z, Zhang LW, Liew KM (2019) Modeling geometrically nonlinear large deformation behaviors of matrix cracked hybrid composite deep shells containing CNTRC layers. Comput Methods Appl Mech Eng 355:753-778. https://doi.org/10.1016/j. cma.2019.06.041

31. Jakubinek MB, Ashrafi B et al (2019) Boron nitride nanotube composites and applications. In: Schulz MJ, Shanov V, Yin Z, Cahay M (eds) Nanotube superfiber materials (second edition) science. Manufacturing, commercialization micro and nano technologies. Elsevier, Amsterdam, pp 91-111. https://doi. org/10.1016/B978-0-12-812667-7.00005-7

32. Ashrafi B, Jakubinek MB et al (2017) Multifunctional fiber reinforced polymer composites using carbon and boron nitride nanotubes. Acta Astronaut 141:57-63. https://doi.org/10.1016/j. actaastro.2017.09.023

33. Odegarda GM, Gatesb TS, Wisea KE et al (2003) Constitutive modeling of nanotube-reinforced polymer composites. Comput Sci Technol 63:1671-1687. https://doi.org/10.1016/S0266 -3538(03)00063-0

34. Zeng QH, Yu AB, Lu GQ (2008) Multiscale modeling and simulation of polymer nanocomposites. Prog Polym Sci 33:191-269. https://doi.org/10.1016/j.progpolymsci.2007.09.002

35. Heshmati M, Yas MH (2013) Free vibration analysis of functionally graded CNT-reinforced nanocomposite beam using Eshelby-Mori-Tanaka approach. J Mech Sci Technol 27:34033408. https://doi.org/10.1007/s12206-013-0862-8

36. Yas MH, Heshmati M (2012) Dynamic analysis of functionally graded nanocomposite beams reinforced by randomly oriented carbon nanotube under the action of moving load. Appl Math Model 36:1371-1394. https://doi.org/10.1016/j. apm.2011.08.037

37. Vallabhan CVG, Das YC (1988) A parametric study of beams on elastic foundations. J Eng Mech Div 114:2072-2082. https://doi. org/10.1061/(ASCE)0733-9399(1988)114:12(2072)

38. Mesut Simsek (2010) Non-linear vibration analysis of a functionally graded Timoshenko beam under action of a moving harmonic load. Compos Struct 92:2532-2546. https://doi. org/10.1016/j.compstruct.2010.02.008

39. Reddy JN (1997) Mechanics of laminated composite plates and shells: theory and analysis, 2nd edn. CRC Press, New York

40. Shu C (2000) Differential quadrature and its application in engineering. Springer, New York

41. Shu C, Du H (1997) Implementation of clamped and simply supported boundary conditions in the GDQ free vibration analysis of beams and plates. Int J Solids Struct 34:819-835. https://doi. org/10.1016/S0020-7683(96)00057-1

42. Nikbakht S, Kamarian S, Shakeri M (2019) A review on optimization of composite structures part II: functionally graded materials. Compos Struct 214:83-102. https://doi.org/10.1016/j.comps truct.2019.01.105

43. Verma V, Jindal VK, Dharamvir K (2007) Elastic moduli of a boron nitride nanotube. Nanotechnology 18:1-6. https://doi. org/10.1088/0957-4484/18/43/435711

44. Mallick PK (2007) Fiber-reinforced composites: materials, manufacturing, and design, 3rd edn. Taylor \& Francis, Dearborn

Publisher's Note Springer Nature remains neutral with regard to jurisdictional claims in published maps and institutional affiliations. 